\title{
Associative memory of structured knowledge
}

\author{
Julia Steinberg ${ }^{1,2,3, *}$ and Haim Sompolinsky ${ }^{2,4}$ \\ ${ }^{1}$ Department of Physics, Harvard University, Cambridge, Massachusetts 02138, USA \\ ${ }^{2}$ Center for Brain Science, Harvard University, Cambridge MA 02138, USA \\ ${ }^{3}$ Joseph Henry Laboratories of Physics, Princeton University, Princeton NJ 08544, USA \\ ${ }^{4}$ Edmond and Lily Safra Center for Brain Sciences, Hebrew University, Jerusalem 91904, Israel
}

\begin{abstract}
A long standing challenge in biological and artificial intelligence is to understand how new knowledge can be constructed from known building blocks in a way that is amenable for computation by neuronal circuits. Here we focus on the task of storage and recall of structured knowledge in longterm memory. Specifically, we ask how recurrent neuronal networks can store and retrieve multiple knowledge structures. We model each structure as a set of binary relations between events and attributes (attributes may represent e.g., temporal order, spatial location, role in semantic structure), and map each structure to a distributed neuronal activity pattern using a vector symbolic architecture (VSA) scheme.

We then use associative memory plasticity rules to store the binarized patterns as fixed points in a recurrent network. By a combination of signal-to-noise analysis and numerical simulations, we demonstrate that our model allows for efficient storage of these knowledge structures, such that the memorized structures as well as their individual building blocks (e.g., events and attributes) can be subsequently retrieved from partial retrieving cues. We show that long-term memory of structured knowledge relies on a new principle of computation beyond the memory basins. Finally, we show that our model can be extended to store sequences of memories as single attractors.
\end{abstract}

Human memory is remarkable in its ability to robustly store and retrieve information with complex and hierarchical structure to guide cognitive processes on many different timescales. This "structured knowledge" can often be conceptualized as sets of associations between discrete events with their contextual attributes. Some concrete examples are, temporal sequences representing events associated to particular times, more general episodic memories representing events associated with particular contexts $[1,2]$, cognitive maps representing spatial environments through landmarks associated with locations [35], and semantic structures in language in which meaning is conveyed through words and their respective roles within the sentence [6-8]. The representation and storage of structured knowledge in long-term memory must be done in a way that allows for its retrieval through partial information, with tolerance for noisy and degraded cues. This is likely facilitated by the distributed nature of the underlying neural representations, which provide an inherent notion of similarity between different representations and a mechanism for learning representations by the the tuning of synaptic weights in a neural network [7, 9-11]. While the utility of distributed representations is clearly beneficial from this perspective, it is still not well understood how to represent associations and relations in neural networks in an efficient and flexible way amenable to higher cognition [12-14].

Several recent studies addressed the contextual modulation of neuronal representations, e.g., by forming "mixed representations" [15] or by gating parts of the network [16]. Several noteworthy proposals have tried to implement more general relational structure in neu-

\footnotetext{
* Correspondence to: jsteinberg@princeton.edu
}

ral networks. A first attempt used the tensor product to create a distributed representation of pairwise relations between discrete items [6]. Subsequently, several Vector-Symbolic Architectures (VSA) were proposed as compressions of the tensor product to avoid the increase in dimensionality of the representation, allowing for the creation of hierarchies of relations in a compact way [1722]. More recently, several architectures for deep or recurrent neural networks have been proposed to promote flexible relational reasoning [23-29]. However, all of these works have primarily focused on working memory, i.e., online tasks of processing incoming structured data and little attention has been given to the challenge of storing and retrieving relational structures in long-term memory.

Storing knowledge structures in long-term memory poses several challenges. While working memory tasks typically process one structure at a time, long-term memory networks must cope with storing multiple structures, such as multiple complex cognitive maps, multiple temporal sequences, or multiple stories. Two generic measures of the efficiency of information storage in recurrent neural networks are their extensive capacity, i.e., the number of stored items scales with the number of neurons in the network [30], and the ability to recall memories from partial cues which have small but significant overlap with the desired memory [31]. Both of these measures can be adversely affected by correlations across memorized patterns. For relational structures, additional correlations may occur due to the presence of objects or contextual attributes in multiple memories, putting additional constraints on the encoding of relational information. In addition to these considerations, models of distributed representation of knowledge structures typically compress the relational structure into a fixed-length distributed vector representation. To compensate for this 
loss of information, "clean-up" mechanisms are invoked. Thus, it is crucial that such mechanisms can be adapted for the task of retrieval of such structures from long-term memory to efficiently store large numbers of relational structures each containing multiple associations.

In this work, we propose a model for associative memory of multiple relational structures by using a quadratic binding scheme to form vector representations of memories consisting of multiple binary relations between items (which we will henceforth denote as pairs of objects and their attributes). While our model is quite general, in most of our work we will use the holographic reduced representation (HRR)[7] VSA scheme for convenience. We show that the binarized versions of these structures can be stored as fixed point attractors in a recurrent neural network. Each structure can be retrieved from the memory network by using a cue which is a structure encoding a subset of the relations in the memorized structure. The holistic nature of this model is highlighted by comparing the storage of temporal sequences in the present model where the entire sequence is stored as a single fixed point, to previous models, where a sequence is stored as a sequence of transitions between multiple fixed points and could not be fully recalled at once [32]. Our model posits that in addition to the network that stores the structures, a Dictionary network stores all individual items (e.g., individual words, familiar objects). We show that the identities of the objects contained in the structure can be decoded faithfully from the retrieved memory by querying the retrieved structure with the appropriate cue as long as a "clean-up" operation is performed to map the noisy estimate of the object to the correct item in the Dictionary. Furthermore, this decoding works well even when the retrieved structure is significantly degraded.

\section{RELATIONAL STRUCTURES}

We model a binary relational structure $S$ as a set of $L$ object/attributes pairs

$$
S=\left\{\left(a_{1}, b_{1}\right), \ldots,\left(a_{L}, b_{L}\right)\right\}
$$

where both objects $a$ and attributes $b$ have embeddings as real vectors representing distributed patterns of activation in a neuronal population, which for simplicity will be assumed to be of the same size $N$. We represent relations between items in a pair $(a, b)$ by a transformation through a pairwise quadratic nonlinearity to a binding vector $g(a, b)\left(\right.$ in $\left.\mathbb{R}^{N}\right)$ representing a distributed pattern of activity in a population of $N$ "binding" neurons. Each component of the binding vector takes the form

$$
g_{k}(a, b)=a^{T} G^{k} b
$$

where each $G^{k}$ is an $N \times N$ fixed binding matrix. The binding operation in Eqn. 2 is a generalized version of a VSA scheme [18] and can be interpreted as a lossy compression of the tensor product binding operation first proposed in [6].

The entire relational structure $S$ is then represented by the vector summation of the individual object/attribute pairs,

$$
\widehat{S}=\sum_{\ell=1}^{L} g\left(a_{\ell}, b_{\ell}\right)
$$

where the vector summation induces a second source of information loss. The representation $\widehat{S}$ is permutation invariant with respect to the index $\ell$ so that the relations within the structure have no particular order. The compressed representations of structures can be used for a variety of computations, such as structure classification. Here we focus on unbinding tasks, in which given $\widehat{S}$ and one of its attributes $b_{\ell}$, we need to estimate its pair $a_{\ell}$. Similar to binding, we assume that the unbinding operation is performed through a quadratic transformation involving the pair $(\widehat{S}, b)$, so that the $k$-th component of the estimator $\hat{a}_{\ell}^{k}$ of $a_{\ell}$ is given by

$$
\hat{a}_{\ell}^{k}=\widehat{S}^{T} F^{k} b, k=1, \ldots, N
$$

where each $F^{k}$ is an $N \times N$ matrix chosen so that the decoding operation is the approximate inverse of the binding operation. In general, the binding and unbinding matrices can be learned and the optimal choice should depend on the nature of the items contained in the dictionary. Here we use a generic set of matrices, a popular choice known as Holographic Reduced Representations (HRR) (Methods).

The final estimate of $a, \tilde{a}$, is computed by comparing the noisy estimate against a Dictionary, i.e., a neural long-term memory system that stores all familiar objects $a_{d}$, using,

$$
\tilde{a}=\arg \max _{d \in \mathcal{D}} d \cdot \hat{a}
$$

This maximum likelihood (ML) decoding is an idealization of a more biologically realistic retrieval of the stored pattern $a$ from a retrieval cue $\hat{a}$ in a long-term associative network that stores all individual Dictionary items. A schematic of the encoding and decoding networks is shown in Fig. 1A.

\section{UNBINDING ACCURACY}

The ML decoding error is given by the probability $P_{\epsilon}$ that the estimator $\hat{a}$ has the largest overlap with an incorrect item in the dictionary. We access the typical decoding performance by considering the case in which $a$ 's and $b$ 's are random vectors with components drawn iid from $\mathcal{N}\left(0, \frac{1}{N}\right)$ and the dictionaries for $a$ 's contains $D$ elements. Then the decoding error is well approximated 
by

$$
P_{\epsilon} \approx \int_{-\infty}^{\infty} D z\left(1-H(-z-\sqrt{\mathrm{SNR}})^{D}\right)
$$

where $D z=\frac{d z}{\sqrt{2 \pi}} e^{-\frac{z^{2}}{2}}$ and $H(z)=\frac{1}{2} \operatorname{erfc}\left(\frac{z}{\sqrt{2}}\right)$. Here SNR is a signal-to-noise-ratio defined in terms of the mean overlap of the estimator $\hat{a}_{d}$ with the correct dictionary item $a_{d}$ and the variance of the overlap with incorrect dictionary item $a_{d^{\prime}}$, i.e.,

$$
\mathrm{SNR}=\frac{\left\langle\hat{a}_{d} \cdot a_{d}\right\rangle^{2}}{\left\langle\left(\hat{a}_{d} \cdot a_{d^{\prime}}\right)^{2}\right\rangle}
$$

where $d^{\prime} \neq d$, and the average is over the Gaussian distributions of the components of $a_{d}$ and $a_{d^{\prime}}$. For full details see Appendix B.

For SNR $\gg 1$, the decoding error can be approximated as

$$
P_{\epsilon} \approx \sqrt{\frac{2}{\pi \mathrm{SNR}}} D e^{-\frac{\mathrm{SNR}}{4}}
$$

To leading order, the SNR for many VSA binding schemes (including HRR) is

$$
\mathrm{SNR} \sim \frac{N}{L}
$$

Eqn. 8 implies that $P_{\epsilon} \ll 1$ as long as $N \gtrsim O(L \log D)$. Hence, for $L \ll N$ accurate decoding requires the size of the dictionary $D$ be at most polynomial in $N$. In this regime, assumed throughout, the size of the dictionary has little effect on performance, which is dominated by the SNR. $P_{\epsilon}$ and the inverse SNR are shown as functions of $L$ in Fig. 1B and C, respectively.

\section{STORING STRUCTURES IN LONG-TERM ASSOCIATIVE MEMORY}

We now consider the long-term memorization of multiple knowledge structures by storing their vector representations in a neural network for long-term Structured Knowledge Associative Memory (SKAM), so that they can be retrieved at a later time from partial cues and subsequently queried to reconstruct individual events. We consider a set of $P$ structures $\left\{S^{1}, \ldots, S^{P}\right\}$ which for simplicity, all consist of $L$ items. We label the set of $\mathrm{L}$ objects and attributes comprising the $\mu$-th structure as $a_{\ell}^{\mu}, b_{\ell}^{\mu}, l=1, \ldots, L$. For each structure, the HRR encoding scheme is used to create a vector representation $\widehat{S}^{\mu}$ from $S^{\mu}$.

To store multiple structures as fixed points in a neural network, the neuronal input-output transformation must be highly non-linear, implying that the stored patterns themselves are limited to the dynamic range of the neurons. As in a standard Hopfield network [30, 31, 33], we
A
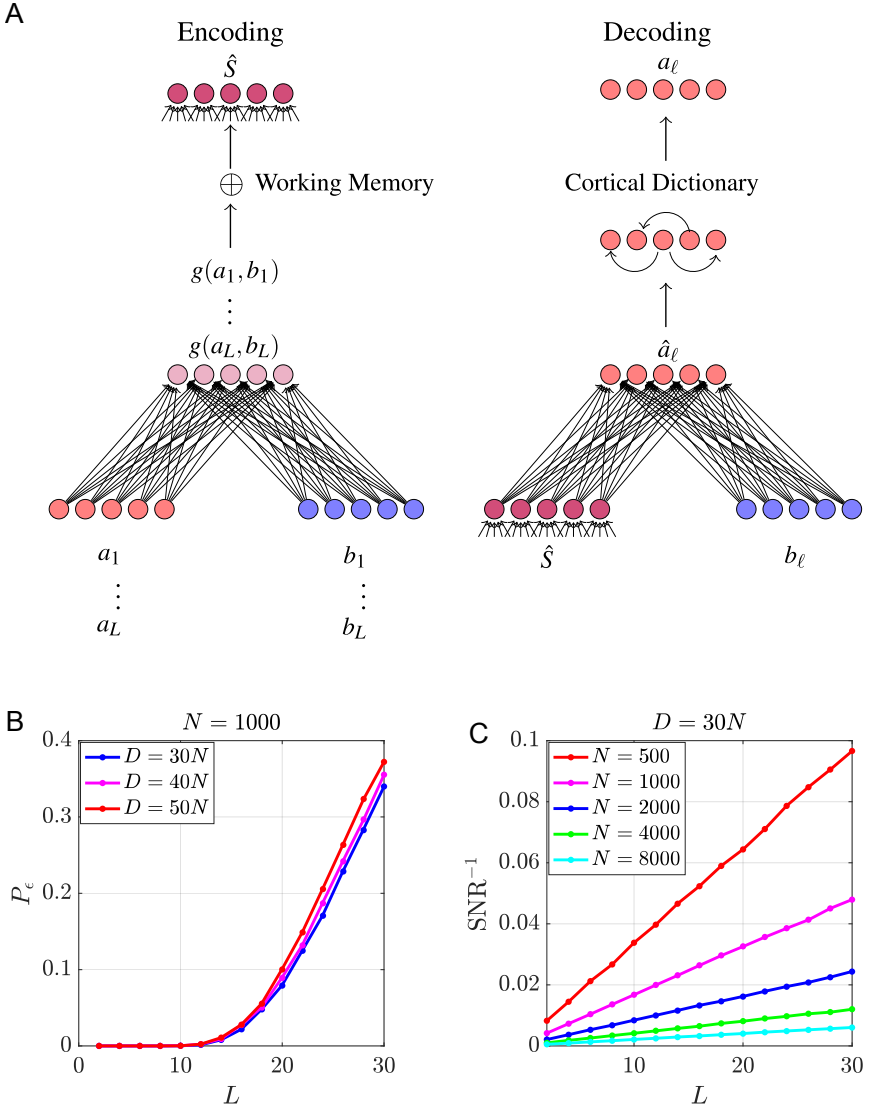

FIG. 1. (A) A schematic of the network used to bind objects/attribute pairs $(a, b)$ to form the knowledge structure $\widehat{S}$ alongside the network used to decode an object $a_{\ell}$ from $\widehat{S}^{\mu}$ by presenting the attribute $b_{\ell}$. (B) The unbinding error $P_{\epsilon}$ shown as a function of structure length $L$ for $N=1000$ for various values of $D$ averaged 10,000 structures (C) $\mathrm{SNR}^{-1}$ shown as a function of $L$ for several values of $N$ averaged over 10,000 structures.

assume neurons are binary \pm 1 variables and the memory patterns, candidates of fixed points of the attractor dynamics are $\sigma^{\mu}=\operatorname{sgn}\left(\widehat{S}^{\mu}\right)$. For a network with $N$ binary neurons, the memory load is defined as $\alpha=\frac{P}{N}$, where $P$ is the number of stored structures.

In general, the associative nature of Hopfield memory networks is expressed in the ability to recall a memorized pattern starting in any initial state which has a sufficiently large overlap with the memorized pattern. If the initial state is within the basin of attraction of the pattern, it will converge to the pattern without errors. In our case, we consider partial cues of a structure, $\widehat{S}$, in the form of a recalling structure $\widehat{S}_{0}$ obtained by binding and subsequent summing any subset $S_{0}$ of the $L$ pairs of binary relations contained in $\widehat{S}$, i.e.,

$$
S_{0}^{\mu}=\sum_{\ell=1}^{L_{0}} g\left(a_{\ell}^{\mu}, b_{\ell}^{\mu}\right)
$$




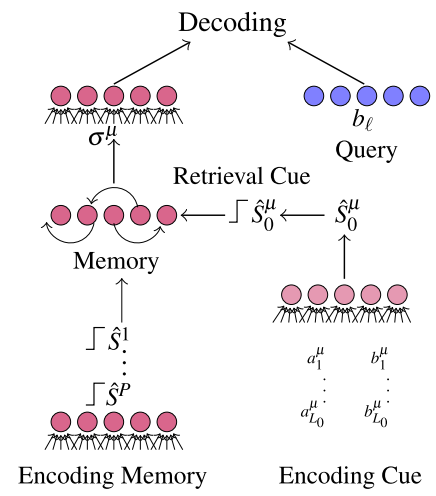

FIG. 2. A schematic of the process of storing multiple binarized structures in a memory network. These structures can be retrieved from memory by encoding a retrieval cue from a subset of the relations in the desired structure as in Eqn. 10 and initializing the network in the state of the binarized cue.

where $L_{0}$ is the number of recalling elements in $\widehat{S}_{0}$ and is assumed to be much less than $L$. The network is then initialized in the state $\sigma_{0}^{\mu}=\operatorname{sgn}\left(\widehat{S}_{0}^{\mu}\right)$ and evolved to a fixed point which, if successful, corresponds to the stored binarized structure $\sigma^{\mu}$. More details on the memory network are given in Methods. A schematic of our model is shown in Fig. 2.

Several learning rules for recurrent networks for associative memory have been studied in the past. Here, for simplicity, we use the Pseudo-inverse learning rule proposed in [34] to train the network. In a Pseudo-inverse network, all structures are perfect fixed points for $\alpha<1$, which is assumed throughout. This allows us to focus on the retrieval cues, since failure to perfectly recall a structure occurs only when the retrieval cue is outside of the basin of attraction of the memorized structure. Similar behavior is observed with the Hebb learning rule for $\alpha$ well below the memory capacity (see Appendix C).

\section{RESULTS}

We evaluate the scheme introduced in the previous section by the ability to accurately extract bound items, say $a_{l}^{1}$ from the structure $\mu=1$ retrieved from the SKAM together with a query in the form of its pair, say $b_{l}^{1}$ (i.e., to successfully perform the unbinding operation as described above). We first quantify performance by the average unbinding error $P_{\epsilon}$ obtained in simulations where structured memories are created from random patterns, stored in memory, retrieved with partial cues $\sigma_{0}^{\mu}=\operatorname{sgn}\left(\widehat{S}_{0}^{\mu}\right)$, and subsequently decoded using the ML "clean-up" operation. We assume all items appearing in memorized structures are stored in dictionaries for objects and for attributes which are then used to decode from the retrieved memory. A schematic of this process is shown in Fig. 2 and full details of the simulations are provided in Methods.
The parameters involved in the performance measure are: network size $N$, memory load $\alpha=P / N$, size of the relational structures and the retrieval cue, denoted as $L$ and $L_{0}$, respectively. We consider the regime where both $N$ and $P$ are very large and the memory load $\alpha \sim O(1)$ $[30,35]$, mainly considering values $\alpha \sim 0.1-0.2$, where the network acts as a good associative memory.

\section{Retrieval of Structured Memories}

We first show numerical results measuring the quality of the retrieved structures in terms of the unbinding error $P_{\epsilon}$ and the SNR of overlaps defined in Eqn. 7. Importantly, in all reported results, the extracted item (and the associated query) comes from pairs that are not part of the cueing structure $S_{0}$. Thus any performance better than chance necessarily involves information extracted by the retrieval from long-term memory. In Fig. 3A we show the dependence of the unbinding error $P_{\epsilon}$ on $L, L_{0}$, and $\alpha$. For comparison, we show $P_{\epsilon}$ for the original structure prior to storage in the memory network, demonstrating that except for small $L$, the dominant contribution to the error comes from retrieving the structure from long-term memory. We also observe that for a fixed $L, L_{0}$, and $\alpha$, the error is suppressed as $N$ increases, in contrast to standard large attractor memory networks where performance depends only on $P / N$. To elucidate this behavior, we replot the results in terms of the $\mathrm{SNR}^{-1}$, i.e., the inverse of the SNR as defined above in Fig. 3B, showing that for each $L_{0}$, there is a critical $L$ above which the SNR of the memorized structures decreases relative to the original $\mathrm{SNR}, \mathrm{SNR}_{0}$ before storage in long-term memory. Note that due to binarization, $\mathrm{SNR}_{0}$ is smaller by a factor of $\frac{2}{\pi}$ relative to the value given in Eqn. 9 . Finally, in Fig. 3C we replot the same results in terms of the inverse of the normalized SNR, SNR/SNR 0 vs., $L / L_{0}$. Note that $\mathrm{SNR}_{0}$ is proportional to $N / L$ so this normalization factors out the "trivial" dependence on $L / N$ from the post retrieval SNR. The figure shows that for a fixed $\alpha$ the normalized inverse SNR depends only on $L / L_{0}$. Finally, we find that the normalized SNR depends only weakly on $N$, suggesting that the main $N$ dependence comes from the linearity of $\mathrm{SNR}_{0}$ in $N$.

\section{Length of Cueing Structure and Memory Basins}

As seen previously in Fig. 3, the performance worsens (and SNR decreases) as $L$ increases, while the converse holds true for $L_{0}$. We find that there is a critical ratio, $l_{c}=\frac{L_{0}}{L}$, defined as the minimum initial cue (relative to the total length), that leads to very small error, essentially equivalent to the error for the original structure.

To understand the origin of $l_{c}$, we note that $\frac{L}{L_{0}}$ specifies the average initial overlap of the retrieval cue with the corresponding memorized structure. This determines whether on average the initial state is within the basin of 
A

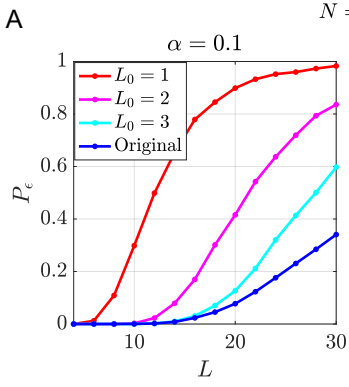

$N=1000$

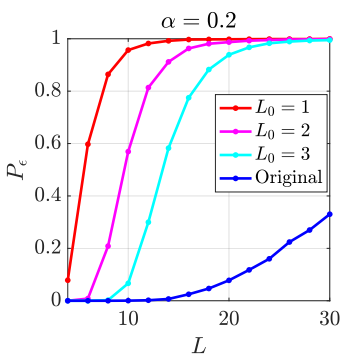

B
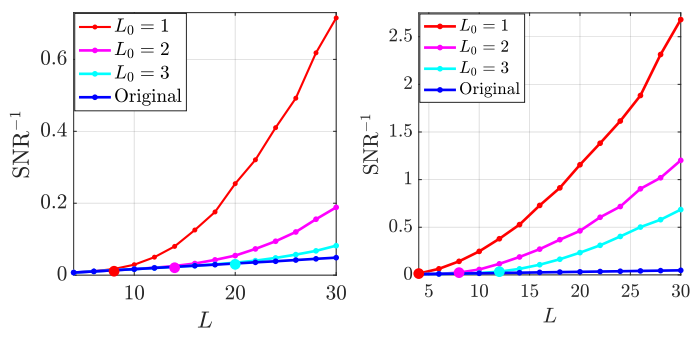

C
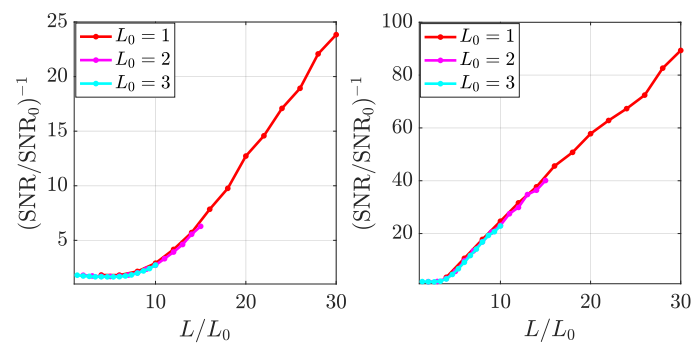

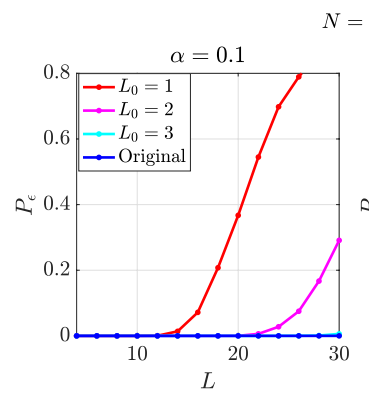

$N=8000$
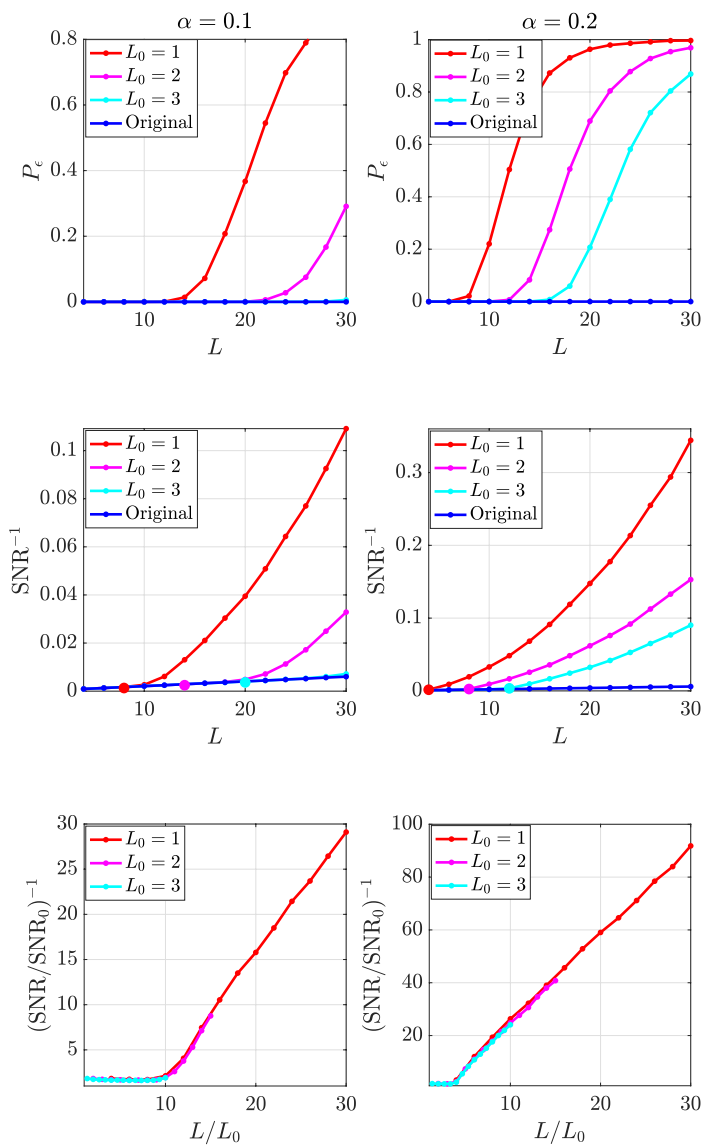

FIG. 3. (A) The decoding error $P_{\epsilon}$ is compared for several values of $L_{0}$ from structures containing $L$ pairs of items for two values of $N$ and two values of $\alpha$. (B) SNR ${ }^{-1}$ v. $L$. For each value of $L_{0}, l_{c}$ is given by the value of $L$ where SNR ${ }^{-1}$ diverges from the line corresponding to the original structure, which is marked for each value of $L_{0}$. $(\mathrm{C})(\mathrm{SNR} / \mathrm{SNR} 0)^{-1} \mathrm{v} \cdot \frac{L}{L_{0}}$ where $\mathrm{SNR}_{0}=\frac{N}{L}$. For all figures, $T=20$ parallel updates are used in memory retrieval and the average is performed over 10,000 memories. The dictionary is fixed to $D=30 N$.

the desired memory, so that the recurrent dynamics will succeed (or fail) in converging to the desired attractor. As the cueing length $L_{0}$ grows, the initial state becomes increasingly likely to be within the basin of attraction of the desired structure, retrieving it with essentially no error. In these conditions, the unbinding operation has the same probability of success as for the original structure.

To determine $l_{c}$ quantitatively, we calculate the relation between $L_{0} / L$ and the average initial overlap $m_{0}$ with the desired structure in the limit of large $N$, yielding

$$
m_{0} \approx \frac{2}{\pi} \arctan \left(\left(\frac{\frac{L_{0}}{L}}{1-\frac{L_{0}}{L}}\right)^{\frac{1}{2}}\right)
$$

which shows how the overlap of the initial state $\sigma_{0}^{\mu}$ with the structure $\sigma^{\mu}$ increases as $L_{0} / L$ is increased. Details are provided in Appendix A.

We define the mean radius of the basins of attraction of the memory fixed points as the maximal fraction of random flips in the memory pattern that will still allow the dynamics to converge to the correct fixed point. This radius is a decreasing function of the load $\alpha$, [34], and is denoted by $R(\alpha)=1-m_{\min }(\alpha)$, where $m_{m i n}(\alpha)$ is the minimal overlap that is still within the basin (on average). Using Eqn. 11, yields

$$
l_{c}(\alpha)=\min \frac{L_{0}}{L} \approx \frac{\tan ^{2}\left(\frac{\pi}{2} m_{m i n}^{2}(\alpha)\right)}{1+\tan ^{2}\left(\frac{\pi}{2} m_{\min }^{2}(\alpha)\right)}
$$

To determine $m_{\min }(\alpha)$ we resort to the Pseudo-inverse model with random binary patterns as memories [34], which is simpler to simulate. Results are shown in Fig. 4A demonstrating a linear increase in the range $\alpha<0.5$. Substituting these results in Eqn. 12 closely predicts the value of $l_{c}(\alpha)$ as shown in Fig. 4B. We conclude that in the regime of interest, where $\alpha \lesssim 0.2$ and $L_{0} / L>l_{c}$, the main source of the decoding error in our model comes from the limitation on good retrieval of the 

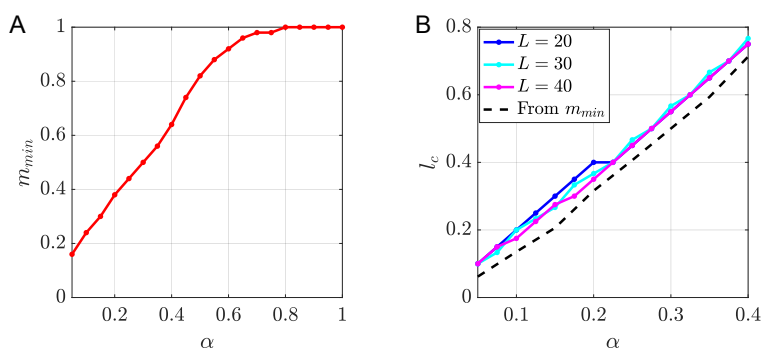

FIG. 4. (A) $m_{\min }$ shown as a function of $\alpha$ for a Pseudoinverse network storing with random patterns. (B) For structured memories, $m_{\min }$ corresponds to a minimum fraction of relations needed obtain a final overlap $m=1$, shown for $L=20,30,40$ along with the value predicted by $m_{\min }$ from the expression in Eqn. 12. $N=1000$ and $T=20$ parallel updates are used in memory retrieval for both figures and the averages are computed over 50 trials.

structure from memory, due to small values of $L_{0} / L$, and not from noise in the original encoding (corresponding to $\left.L_{0} / L>l_{c}\right)$.

\section{Retrieval Outside Memory Basins}

Naively, one would expect that for $L_{0}<l_{c} L, P_{\epsilon}$ will be very large due to the accumulation of errors in the retrieved structure, which is a fixed point outside the memory basin. However, as shown in Fig. 4, this is not case. Surprisingly, the decoding performance is well below chance for substantial range of values of $L$, even when $L \gg L_{0} / l_{c}$. This observation can be explained by two scenarios: (1) the actual basins fluctuate in their shape so that for some structured memories, initial states may converge to the memory fixed point even if they are outside the mean basin radius; (2) initial states outside the true memory basin converge to fixed points with significant overlap with the structured memory. To test these scenarios, we measured the empirical distributions $p(m)$ where $m$ is the overlap between the fixed point and the desired structure, obtained from histograms of overlaps for several values of $L>\frac{L_{0}}{l_{c}}$, shown in Fig. 5 . We find that $p(m)$ is bimodal and takes the general form

$$
p(m)=\left(1-p_{1}\right) p_{m<1}(m)+p_{1} \delta(m-1)
$$

where $p_{1}$ is the probability that a structure is perfectly retrieved from memory and $p_{m<1}(m)$ corresponds to the distribution of $m$ for imperfectly retrieved memories.

The peak at $m=1$ corresponds to trajectories converging at the target memories, despite the fact that the initial overlap $m_{0}$ is outside the mean basin radius, indicating non-spherical basin shape. The second mode, peaked at $0<m<1$ results from trajectories that converged to a fixed point outside the basin but with a significant residual overlap with the memory.

As noted in $[34,35]$, the shape of the distribution $p(m)$
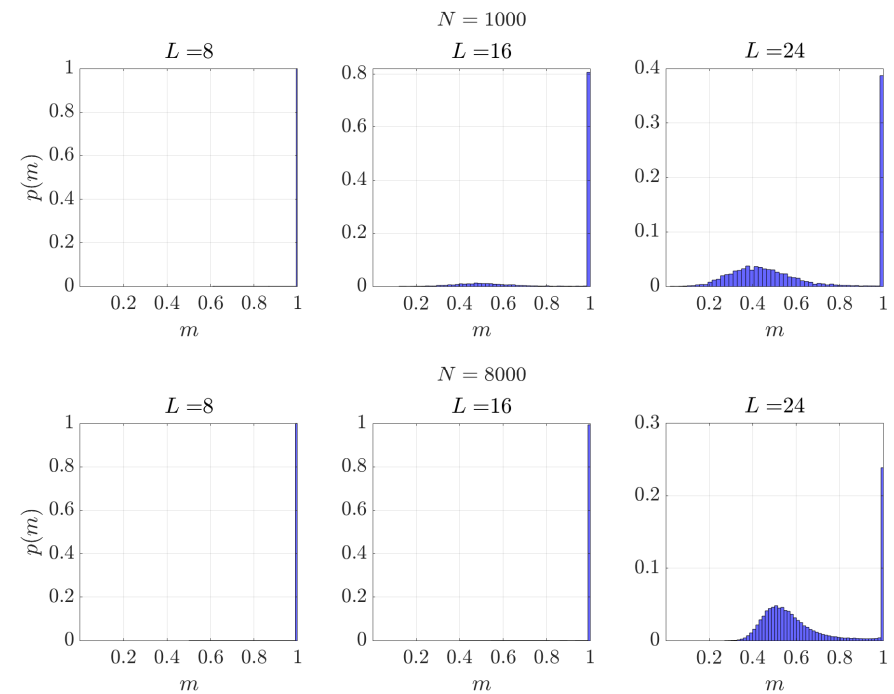

FIG. 5. Histograms of the final overlaps $m$ for various values of $L$ for $\alpha=0.1$ and $L_{0}=2$ and 100 trials. $T=20$ parallel updates are used to retrieve memories. The top panel shows $N=1000$ and bottom shows $N=8000$.

is sensitive to finite size effects. To analyze these effects, we calculate $p(m)$ for different sizes in a standard Pseudo-inverse model where the initial overlap $m_{0}$ can essentially be varied continuously. For $m_{0}>m_{\min }(\alpha)$ almost all trials converge to the memorized pattern. For $m_{0}<m_{\min }(\alpha) p(m)$ is bimodal and has a similar shape to those of Fig. 5 (for the corresponding values of $m_{0}$, and $N$, see SI, Fig. 14). We characterize the shape of the distribution by the probability of $m=1, p_{1}$, the width of the lower $m$ mode, $\sigma_{m}$ and the mean of that mode, $\bar{m}$. Results are shown in Fig. 6A, for several values of $N$ and two values of $\alpha$. We find that for large $N, p_{1}$ approaches a step function changing from zero to one as $m_{0}$ crosses $m_{\min }(\alpha)=1-R(\alpha)$. Near this transition, it can be approximated as

$$
p_{1} \sim \frac{1}{1+e^{-\sqrt{N}\left(m_{0}-m_{\min }(\alpha)\right)}}
$$

indicating that $p_{1}$ converges to a step function exponentially fast with $\sqrt{N}$. In addition, $\sigma$ is very small outside the narrow transition regime of $m_{0}$ and shrinks to zero everywhere as $1 / \sqrt{N}$. From this, we conclude that for $N \rightarrow \infty, p(m)$ becomes a $\delta$ function, which is either located at $m=1$ for $m_{0}>m_{\min }$ or at a smaller value $\bar{m}$ which increases smoothly with $m_{0}$, starting from zero and reaching 1 as $m_{0}$ increases from zero to $m_{\min }$. Thus in large networks, the basins have a roughly spherical shape, such that virtually all initial conditions with $m_{0}$ larger than the mean minimal overlap converge to the memory, and all initial conditions with smaller $m_{0}$ converge to fixed points with nonzero partial overlap, $\bar{m}$. We also find that the rate of increase of $\bar{m}$ with $m_{0}$ depends 
on load $\alpha$, such that

$$
\bar{m}\left(\alpha, m_{0}\right) \sim f(\alpha) m_{0}
$$

for $m_{0}<m_{\min }(\alpha)$ and $m(\alpha)=1$ otherwise. We find for $\alpha \lesssim 0.3, f(\alpha)>1$ shown in Fig. $6 \mathrm{C}$, implying that the final overlap with the retrieved structure is significantly larger than the initial overlap $m_{0}$, shown by the black line in Fig. 6A, even far outside the basin for the structure.
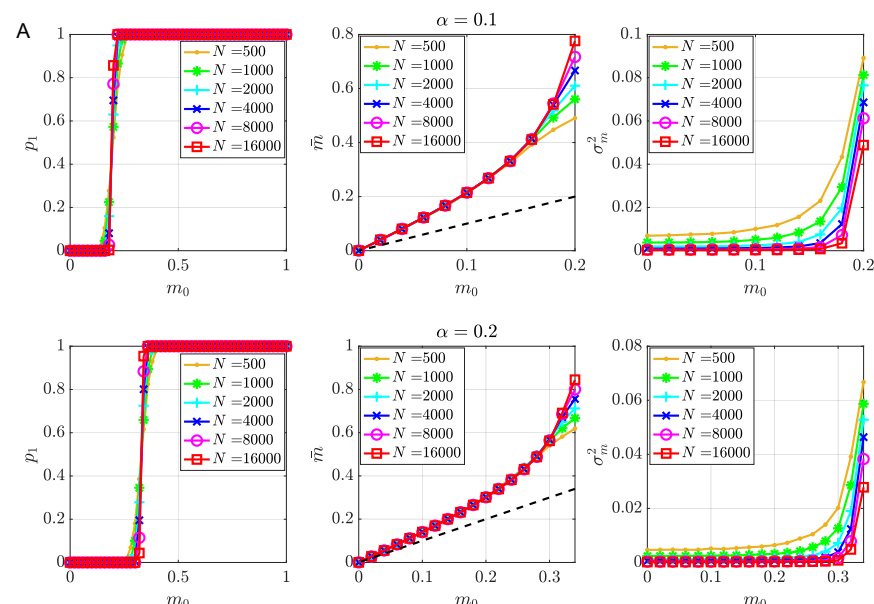

B
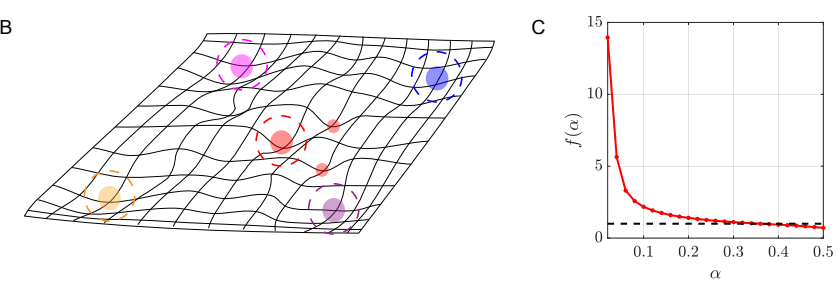

FIG. 6. (A) $p_{1}, \bar{m}$, and $\sigma_{m}^{2}$ are shown as functions of the initial overlap $m_{0}$ for several values of $N$ for pseudo-inverse networks storing random memories with memory loads $\alpha=0.1$ and $\alpha=0.2$. (B) A visual of the energy landscape. The large filled circles are stored memories and the dashed circles denote their basins of attraction. The two small red circles are fixed points outside of the basin of attraction of the red memory which still lead to a large enough overlap for accurate decoding when $1 \gg N / L$. (C) $f(\alpha)$ (Eqn. 15) obtained from simulations of a pseudo-inverse network storing random memories of size $N=8000 . \quad T=20$ parallel updates are used for memory retrieval and averages are performed over 50 trials.

\section{SNR of Retrieved Structures Outside the Basin}

We now use the preceding results to estimate the SNR for $L_{0} / L \ll l_{c}$, i.e., when the initial state is well outside the memory basin. First, we argue that the SNR of unbinding from a noisy state with overlap $m<1$ with the true structure, should be roughly,

$$
\operatorname{SNR}(m) \sim \frac{2 c}{\pi} \frac{m^{2} N}{L}
$$
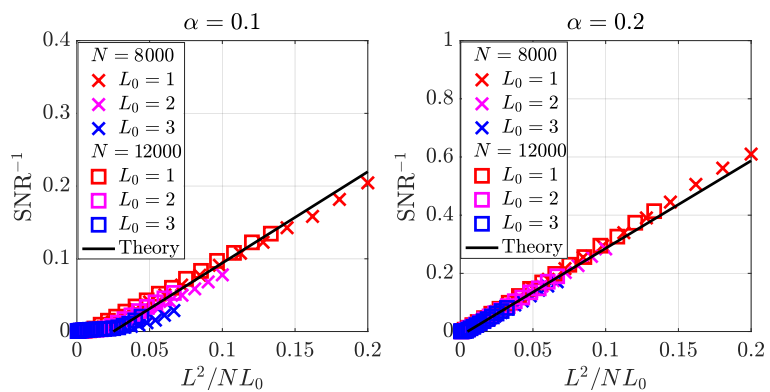

FIG. 7. $\mathrm{SNR}^{-1} \mathrm{v} . L^{2} / N L_{0}$ shown for $N=8000$ and $N=12000$ and for several values $L_{0}$ as an average over items contained in 10,000 memories shown for $\alpha=0.1$ and $\alpha=0.2$. The black line is obtained from Eqn. 17 with $c=0.65 . T=20$ parallel updates are used for memory.

where, as before, the factor of $\frac{2}{\pi}$ comes from binarization and $c \approx 0.65$ accounts for the fact that part of the overlap $m$ is contributed by the initial cueing structure $S_{0}$ and is more concentrated around the relations contained in the retrieval cue. Using Eqns. 11 and 15 we arrive at

$$
\mathrm{SNR}_{0} \sim \frac{N L_{0}}{L^{2}} \frac{8 c}{\pi^{3}} \frac{f(\alpha)^{2} L_{0}}{L}, L_{0} \ll L
$$

These predictions are verified in Fig. 7 for two values of $\alpha$. These results summarize the rich behavior of associative memory of structured knowledge. In contrast to standard memory functions, here the performance depends not only on number of patterns per neuron $\alpha$ but also on the network size $N$, structure length $L$, and cueing length $L_{0}$ through the SNR, in addition to the structured memory load $\alpha$. The key difference is the fact that in structured memories, the criterion for success is not limited to convergence to the target memory; even if the target memory is only partially retrieved, the underlying memorized relations can be still be retrieved faithfully using the semantic memory. The other unique feature is that the well-known property of pattern completion is realized here by a sub-structure of length $L<L_{0}$, (in addition to the standard random initial condition).

\section{ALTERNATIVE LEARNING RULES}

So far we have considered storing structured memories in recurrent neural networks in which the synaptic weights are set via the Pseudo-inverse rule. This learning rule fully decorrelates linearly independent memories so that each memory is a perfect fixed point for $\alpha<1$. However, the Pseudo-inverse rule is both non-local and non-incremental, thus it is unlikely to be biologically implemented in a straightforward manner. We now consider the storage of structured memories with two learning rules that are both local and incremental. The first is the Hebb rule and the second is the Storkey rule proposed in [36-38] (see Eqns. (27)-(29) in Methods and 
Appendix C). The Storkey rule can be viewed as a biologically plausible approximation to the Pseudo-inverse rule for small $\alpha$ [37]. As a result, the storage capacity of this rule $\alpha_{c} \approx 0.4$ is significantly higher than the Hebb rule $\left(\alpha_{c} \approx 0.14\right)$ and the basins of attraction are larger and more even across different memories [38].

Our previous analysis applies to these learning rules as well. In general, we find that outside of the basin of attraction, $\bar{m}$ scales linearly with $m_{0}$ for all three learning rules and takes the form in Eqn. 15 where the coefficient $f(\alpha)$ depends on the learning rule. More details are given in Appendix C.

We compare $P_{\epsilon}$ for structures stored using the Hebb, Storkey, and Pseudo-inverse rules in Fig. 8A. In general, we find that for low values of $\alpha \lesssim 0.3$, the error of decoding from memories stored via the Storkey rule is very similar to the Pseudo-inverse rule. Both are significantly lower the error obtained when decoding from memories stored using the Hebb rule. This suggests that the Storkey learning rule sufficiently decorrelates the different structures to allow for both efficient storage and retrieval of structured knowledge.
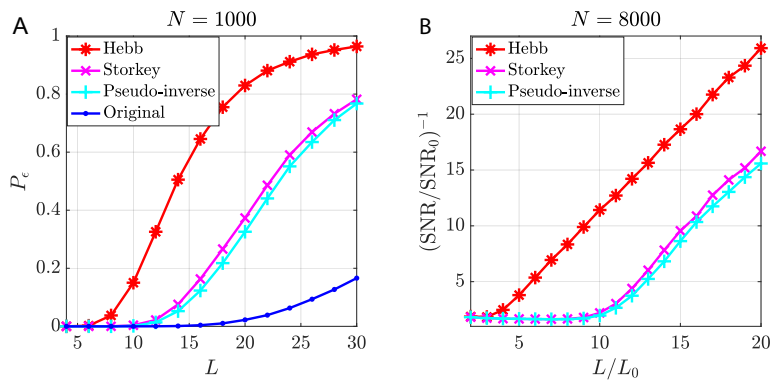

FIG. 8. (A) Comparison of the decoding error $P_{\epsilon}$ for structures of size $N=1000$ stored using the Hebb rule, Storkey rule, and Pseudo-inverse rule at memory load $\alpha=0.1$ and retrieved from memory with a partial cue of length $L_{0}=2$. The $P_{\epsilon}$ for the original structure is also shown for comparison and the size of the dictionary is fixed to $D=30 N$. (B) $\left(\mathrm{SNR} / \mathrm{SNR}_{0}\right)^{-1} \mathrm{v}$. $L / L_{0}$ shown for $N=8000$. $T=20$ parallel updates are used for both figures and the is average is performed over 20,000 memories.

\section{STORAGE AND RETRIEVAL OF SEQUENCES}

\section{Storing Sequences as Binary Structures}

We now extend the results of the previous sections to temporal sequences. Such sequences can be modelled as structures in several ways. One possibility is to bind each event in the sequence with its temporal order in the sequence, as was done in the Temporal Context Model of free recall of lists [2]. Here we use temporal proximity as the contextual cue. Namely, we interpret a sequence as a set of binary associations between temporally proximal events. Thus, a temporal sequences of length $L$, $\left(a_{1}, a_{2}, \ldots, a_{L}\right)$ can be represented as a structure of the form

$$
S=\left\{\left(a_{1}, a_{2}\right),\left(a_{2}, a_{3}\right), \ldots,\left(a_{L-1}, a_{L}\right)\right\}
$$

As before, the entire sequence $S$ is then represented by a vector $\widehat{S}$ of size $N$ given by

$$
\widehat{S}=\sum_{\ell=1}^{L-1} g\left(a_{\ell}, a_{\ell+1}\right)
$$

Decoding an episode at a particular time, say $a_{\ell}$, is performed through an unbinding operation with a query by the preceding event, $a_{\ell-1}$. Starting from a query by $a_{1}$, the entire sequence can be unfolded through a sequence of queries. Because each event appears in two binary relations, we need to use an asymmetric binding operation so that $g(a, b) \neq g(b, a)$. Within HRR, this can be accomplished by switching the binding and unbinding operations [39].

As before, we consider the case in which all items being decoded are contained in a dictionary $\mathcal{D}=$ $\left\{a_{1}, a_{2}, \ldots, a_{D}\right\}$, so each decoding step involves a cleanup of the decoded item before preceding to decode the next item from the sequence. A schematic of this process is shown in Fig. 9A.

The binarized versions of the structures representing each sequence are stored for long-term memory in a recurrent neural network with synaptic weight matrix determined via the Pseudo-inverse rule. The cueing structure $\widehat{S}_{0}$ consists of the first relation $\left(a_{1}, a_{2}\right)$, so the overlap of $\widehat{S}_{0}$ with the stored sequence $\widehat{S}$ is closely approximated by Eqn. 11 with $L_{0}=2$. Alternatively, the first item $a_{1}$ can be used as a retrieval cue if it is added to the representation $\widehat{S}$ in Eqn. 19. We are primarily interested in the ability to reconstruct the entire sequence after it is retrieved from memory.

\section{Retrieval of Sequences from Long-term Memory}

Because of the sequential nature of the decoding of sequences in our model, the decoding error is accumulating and performance of unbinding of an event depends on its position in the sequence (relative to the cued events). In Fig. 9B, we show the decoding error $P_{\epsilon}$ at each position along sequences encoded by Eqn. 19 for sequences of different length $L$. Since the SNR of the overlap with the correct item along each position in the sequence depends on $L$, the length of the sequence limits the accuracy of decoding at all positions along the sequence. Nevertheless, for low memory loads and moderately long sequences, the accumulated error is small. An interesting outcome of this mode of recall is the accumulation of errors as the recall sequence advances. This would give rise to correlations between probabilities of recall that decay as a function of the temporal lag between the events, consistent 
A
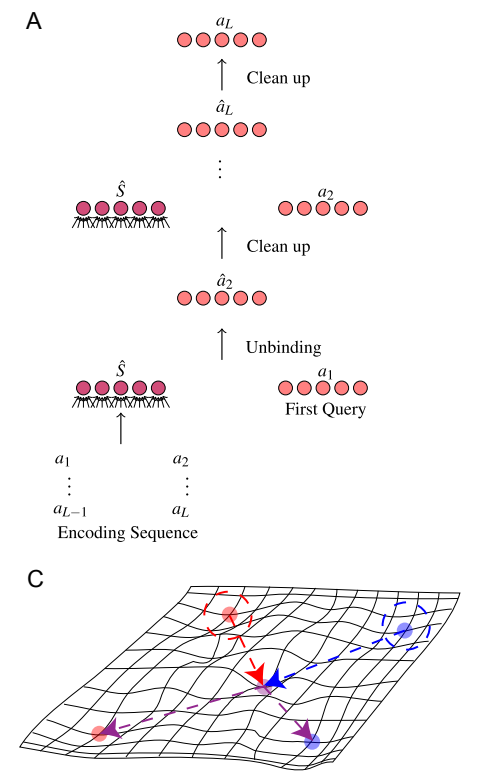

E

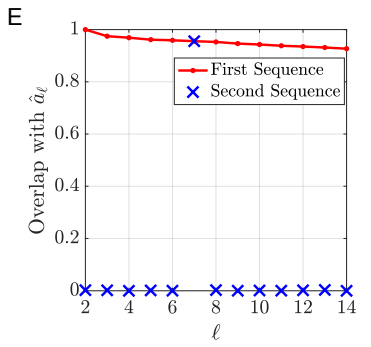

B

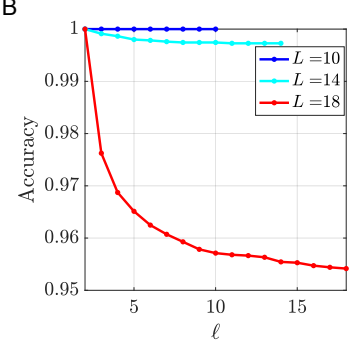

D

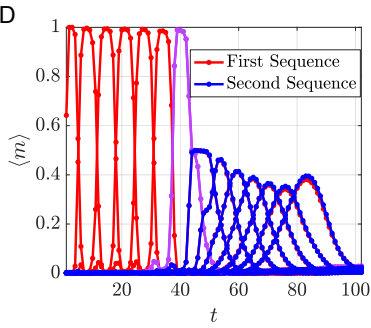

$\mathrm{F}$

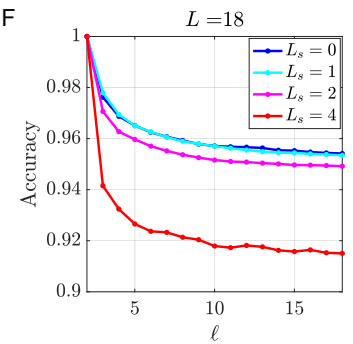

FIG. 9. (A) A schematic of the decoding scheme for sequences. (B) Decoding accuracy as a function of sequence position $\ell$ along sequences of length $L$ retrieved from memory shown for several values of $L . N=2500, \alpha=0.05$, and the average is computed over 100 trials. (C) A schematic of the energy landscape for two temporal sequences containing a single common element which are encoded as sequences of attractors using the scheme in [32] (discussed in Appendix D). (D) The average overlap of the network state $\langle m\rangle$ with each attractor in the sequence is shown as a function of retrieval time for a network of size $N=1000$ storing two sequences containing a single element in common. The parameters $\tau=8$ and $\lambda=2.5$ are used and the average is computed over 1000 trials. (E) The average overlap of the estimator $\hat{a}_{\ell}$ (normalized by $\left\langle\hat{a}_{2} \cdot a_{2}\right\rangle$ ) with the correct dictionary item at each position in the sequence for a network of size $N=1000$ storing two sequences containing a single element in common. The average is computed over 10,000 trials. (F) Decoding accuracy as a function of sequence position $\ell$ for $P=\alpha N$ sequences for which each neighboring sequence contain $L_{s}$ common elements with the previous one. $N=2500, \alpha=0.05$ and the average is computed over 100 trials.

with observations [2]. This behavior was previously explained by positing that proximal temporal context vectors are correlated. In our model, these correlations are a natural consequence of the fact that the proximal events

serve as temporal context cues.

The present scheme of long-term storage of sequences as single fixed points overcomes a key disadvantage of previous attempts at storing multiple temporal associations in attractor neural networks. In previous models of sequential memory [32], all of the patterns contained in the sequences are stored as separate attractors in one network and the sequences themselves are encoded in a time-delayed synaptic plasticity rule that associates each pattern with its next pattern in the sequence, illustrated in Fig. 9C and reviewed in Appendix D. Because of the Markovian nature of the synaptic plasticity, retrieval will fail if multiple sequences share the same event, as illustrated in Fig. 9D. By contrast, in the present model, the entire sequences are stored and retrieved as separate "holistic" attractors. Thus, as long as the retrieval cue is unique to a single sequence, it will retrieve it unambiguously and the subsequent unfolding of the sequence by the unbinding network will be immune from interference with other sequences. To demonstrate this, we consider $P$ sequences $S^{\mu}$ with $\mu=1, \ldots, P$ of length $L$ where neighboring sequences $S^{\mu}$ and $S^{\mu+1}$ share $L_{s}$ events in common. An example of storing two sequences with an overlapping event is shown in Fig. 9E demonstrating the successful retrieval of the entire sequence despite the presence of an overlapping state (compare with Fig. 9D). Figure 9F shows that sequences can be faithfully retrieved even with multiple common states, up to the point where the basins of attraction of individual sequences shrink due to the large overlap between them.

Finally, it is interesting to compare the the memory capacity of the two models. In the sequence attractor model, the maximal number of stored sequences of length $L$ is $P<\alpha_{c} N / L$ since the network stores $P L$ states. In contrast, in the present model, since only $P$ attractors are stored, the capacity of storage is $P<\alpha_{c} N$. Nevertheless, for a successful unfolding of the sequence we need $P L / N$ to be bounded (for a fixed $L_{0} / L$ ) due to the noise in the unbinding operation. A potential disadvantage of the current model is the need to devote additional memory resources to store the individual events in a Dictionary. On the other hand, the Dictionary can be used for multiple other cognitive tasks aside from the retrieval of these sequences. Another flexibility in the separation of the retrieval of the neural representation of the sequence from the subsequent reconstruction of individual events, is the fact that, for some tasks, the agent may not need to access the full detail of the sequence, for instance in tasks that requires distinguishing between one episode and another one. Such tasks may not need to rely on the full unbinding of the sequences.

\section{DISCUSSION}

In summary, we have proposed and analyzed a model demonstrating how multiple knowledge structures, i.e., sets of relations between pairs of items, can be repre- 
sented, stored, and retrieved in Hopfield type recurrent neural network models of long-term memory. Our model hypothesizes that the entire set of relations is encoded through binding operations, summation and binarization, in a single pattern of activity in a neuronal population, which is then stored as fixed point in the recurrent network. Retrieval of relational information from longterm memory, consists in our model of two stages: first, retrieval of the desired fixed point, and subsequent unbinding to uncover individual relations with the aid of a separate memory system, the Dictionary.

We also show that this scheme can be used to model long-term memory of temporal sequences by creating structure vectors for sequences of temporally associated items and store in a recurrent network compressed versions of the sequences as fixed points. Sequence recall consists of retrieval of the "sequence" fixed point, and subsequent unfolding of the stored events through a sequence of unbinding operations. In this application we have also demonstrated that our model for storing structure vectors in long-term memory is not very sensitive to the presence of a partial overlap between different structures.

Our analysis suggests the success of this long-term memory system depends not only on the memory capacity of the attractor network but also very crucially on the properties of the memory basins of attraction and the landscape in the surrounding "terrain", such as the degree of overlap between "spurious" states outside the basins with the target fixed point (inside it). For this reason, a learning rule that decorrelates memories and yields smoother basins is clearly superior, as shown in Fig. 8. Due to the dense distributed nature of the binding scheme employed here (HRR), we have not studied the effect of pattern sparsity on the long-term memory system [40]. It would be interesting to explore the sparsity effect in sparse binding schemes [41-44].

We close by briefly discussing three important aspects of this work which have the most immediate phenomenological implications. The first is the implementation of binding through multiplicative interactions between neurons. We note that the binding operation can be written as $g_{k}(a, b)=a^{T} b^{\prime}$ where $b^{\prime}=G^{k} b$. The last term can be implemented by propagating the pattern $b$ through a synaptic matrix $G^{k}$. Finally the dot product between $a$ and $b^{\prime}$ can be implemented through multiplicative interactions in biological neurons [45]. Previously, several mechanisms have been proposed to facilitate multiplicative interactions among neurons including dendritic gating [46], quadratic firing rate response [47], and shortterm synaptic plasticity. Short-term plasticity comprises a variety of synaptic processes that modulate synaptic efficacy in a transient, activity dependent manner [48]. These processes occur on timescales ranging from milliseconds to minutes [49] and are thought to mediate higher cognitive functions like attention and working memory. More recently it has been suggested that "fast weights" in artificial neural networks may serve as an analogy to short-term plasticity in the brain [50] with connections to linear transformers [51].

A second key aspect of our model is the existence of neuronal populations representing entire relational structures in long-term memory as persistent patterns of activity displaying the "holistic context" of each structure. This system interacts with a working memory system which executes the dynamics of retrieving details of the stored relations. We have not addressed the interesting question of the mechanism by which a stream of experiences is segmented into a sequence of discrete events [52], or more generally, the mechanism that segments complex environments into a discrete sets of bound items. In particular, our model of long-term memory of sequences predicts that the retrieval of a temporal sequence is associated with a persistent pattern of activity (representing the context of the entire sequence) in addition to sequential dynamics involving the dynamic interaction between working and long-term memory. This can be tested in recordings of neuronal activity during recall of sequences in the hippocampus and in songbirds.

Finally, as mentioned above, our framework of storage and retrieval of relational knowledge structures in longterm memory relies on the existence of a complementary long-term memory system, the "Dictionary", which stores the individual building blocks comprising the relational knowledge. It is tempting to identify these two complementary memory systems as representing episodic memory (the relational system) and semantic memory (the Dictionary) [53], although we emphasize that in the present context, semantic memory does not necessarily require language and presumably exists in other species as well. The synergy of these two "complementary" memory systems results in an associative memory system with both the capacity and flexibility to store and faithfully represent complex knowledge structures in long-term memory. Adapting this framework to further explain empirically observed phenomena in memory will require adherence to known biological properties of hippocampal representations as well more explicit models of both the Dictionary and the working memory system in which binding and unbinding occurs.

\section{MATERIALS AND METHODS}

\section{Holographic Reduced Representation}

$\operatorname{HRR}[8,54]$ is a commonly used VSA scheme with fixed forms for the binding and unbinding matrices in Eqns. 2 and 4 . The binding operation $g$ is given by the circular convolution operation of the vectors $a$ and $b$ where

$$
g_{k}=\sum_{j=0}^{N-1} a_{j} b_{k-j}, k=0, \ldots, N-1
$$


and all subscripts are defined modulo $N$. The circular convolution operation is both associative and commutative. The corresponding decoding operation $\phi$ is realized through circular correlation of the two vectors $\widehat{S}$ and $b$ where

$$
\hat{a}_{k \ell}=\sum_{j=0}^{N-1} \widehat{S}_{j} b_{j+k, \ell}
$$

We see from comparing Eqns. 2 and 4 with Eqns. 20 and 21 that HRR corresponds to the following choice for the encoding and decoding matrices

$$
\begin{aligned}
G_{i j}^{k} & =\delta_{k, j+i} \\
F_{i j}^{k} & =\delta_{k, j-i}
\end{aligned}
$$

The commutativity of the encoding operation implies that HRR encoding is commutative. To represent noncommutative asymmetric relations, we can simply exchange the binding and unbinding operations i.e., binding with circular correlation and unbinding with circular convolution [39]. The full details of the statistics of decoding for HRR is given in Appendix A.

\section{Memory Network}

Throughout this work, we consider Hopfield type recurrent neural networks with binary neurons. The state of the network at time $t, \sigma(t)$ is given by the update rule

$$
\sigma_{i}(t)=\operatorname{sgn}\left(\sum_{j} J_{i j} \sigma_{j}(t-1)\right)
$$

where updates are done either in series or in parallel. For simplicity, parallel updates are used for the figures in the main text, but we show in Appendix $\mathrm{C}$ that the results are qualitatively similar for serial updates.

Given a set of memories $\sigma^{\mu}$, the synaptic weight matrix $J_{i j}$ must be chosen so that each of the memories is a fixed point of the dynamics in Eqn. 24. There are several different learning rules which can accomplish this. Mainly, we consider the Pseudo-inverse rule [34] with synaptic weight matrix given by

$$
J_{i j}=\frac{1}{N} \sum_{\mu, \nu=1}^{P} \sigma_{i}^{\mu} C_{\mu \nu}^{-1} \sigma_{j}^{\nu}, J_{i i}=0
$$

where the pattern overlap matrix $C_{\mu \nu}$ is defined as

$$
C_{\mu \nu}=\frac{1}{N} \sum_{i=1}^{N} \sigma_{i}^{\mu} \sigma_{i}^{\nu}
$$

We also consider the Hebb rule given by

$$
J_{i j}=\frac{1}{N} \sum_{\mu=1}^{P} \sigma_{i}^{\mu} \sigma_{j}^{\mu}, J_{i i}=0
$$

and the Storkey rule [36-38] with $J_{i j}$ given by

$$
\begin{aligned}
J_{i j}^{\mu} & =\frac{N+1}{N-1} J_{i j}^{\mu-1}+\frac{1}{N-1}\left(\sigma_{i}^{\mu} \sigma_{j}^{\mu}-\delta_{i j}\right) \\
& -\frac{1}{N-1}\left(\sigma_{i}^{\mu} h_{j}^{\mu}+\sigma_{j}^{\mu} h_{i}^{\mu}\right) \\
J_{i i}^{\mu} & =0
\end{aligned}
$$

where,

$$
h_{i}^{\mu}=\sum_{k=1}^{N} J_{i k}^{\mu-1} \sigma_{k}^{\mu}
$$

These learning rules differ in their capacity and the average size of the basins of attraction for memories at a given memory load $\alpha$, further discussed Appendix C.

\section{Simulations}

We simulate the memory storage, retrieval, and decoding processes by creating dictionaries of objects and attributes $\mathcal{D}^{a}$ and $\mathcal{D}^{b}$ where $a$ 's and $b^{\prime}$ s are random vectors with components drawn iid from a Gaussian distribution i.e. $a_{i} \sim \mathcal{N}\left(0, \frac{1}{N}\right), b_{i} \sim \mathcal{N}\left(0, \frac{1}{N}\right)$. The size of these dictionaries is fixed to $D=L_{\max } N$, where $L_{\max }$ is the size of the largest structure being considered. In Fig. 1B we show the decoding error for several values of $L_{\max }$ and for Figs. $1 \mathrm{C}, 3$ and 8 we set $L_{\max }=30$. We then use a subset of the dictionaries to create $P$ knowledge structures with vector representations given by HRR encoding. These structures are then point-wise binarized and used to compute the synaptic weight matrix using the Pseudo-inverse rule unless otherwise stated. Since the encoding of the structures induces a similarity with individual relations $g(a, b)$ rather than with $a$ or $b$ individually, we find that the same set of attributes $\left\{b_{1}, b_{2}, \ldots b_{L}\right\}$ can be the same across several or all of the different knowledge structures while retaining the ability to decode the corresponding object $a_{\ell}^{\mu}$ from retrieved structure $\sigma_{r}^{\mu}$. Hence, we consider the case in which the same attributes are used in each structure i.e. $b_{\ell}^{\mu}=b_{\ell}$.

We test the performance of the memory network by initializing the network in the state $\sigma^{\mu}(0)=\sigma_{0}^{\mu}=\operatorname{sgn}\left(\widehat{S}_{0}^{\mu}\right)$ for each memory $\mu=1, \ldots, P$ where $S_{0}^{\mu}=\left\{\left(a_{\ell}^{\mu}, b_{\ell}^{\mu}\right)\right\}_{\ell=1}^{L_{0}}$ is the subset of $L_{0}$ relations used to create a retrieval cue. We then evolve the network for $T$ parallel updates, denoting the attractor reached by the network as $\sigma_{r}^{\mu}=\sigma^{\mu}(T)$ , i.e., the retrieved state starting from partial cue of the $\mu$-th structure. We define $m^{\mu}$ as the overlap between $\sigma^{\mu}$ 
and $\sigma_{r}^{\mu}$ i.e.

$$
m^{\mu}=\frac{1}{N} \sum_{i=1}^{N} \sigma_{i}^{\mu} \sigma_{r i}^{\mu}
$$

For each retrieved structure $\sigma_{r}^{\mu}$, we use $b_{L}^{\mu}$, corresponding to a relation not contained in the initializing structure, to obtain an estimate $\hat{a}_{L}^{\mu}$ for $a_{L}^{\mu}$, which then identified with the Dictionary element with which it has the highest overlap.

The Pseudo-inverse rule ensures that the basins of attraction for different structures are essentially identical regardless of potential differences in the overlap between different structures. In simulations, this allows us to consider each structure as an independent trial. The fraction of trials in which $a_{L}^{\mu}$ is incorrectly decoded from $\sigma_{r}^{\mu}$ provides an empirical estimate of the decoding error $P_{\epsilon}$. We also construct an empirical SNR from Eqn. 7. Finally, we measure $m^{\mu}$ for each structure (Eqn. 30) to obtain an empirical distribution $p(m)$ where the overlaps $m$ are calculated for each memory in a trial and accumulated over many trials. The distribution $p(m)$ does not appear to change if measured over multiple trials with different patterns or for multiple patterns within the same trial, which further supports the ability to consider each structure as a separate trial. The distribution $p(m)$ is a statistical measure of the retrieval quality for structures of fixed size $L$, memory load $\alpha$, and retrieval cue length $L_{0}$.

\section{Acknowledgements}

We thank Tankut Can, Naoki Hiratani, Mikhail Katkov, Kamesh Krishnamurthy, Andrew Saxe, Nimrod Shaham, and Misha Tsodyks for fruitful discussions. J.S. acknowledges support from the NSF through the Center for the Physics of Biological Function (PHY-1734030) and computational resources from the Princeton Research Computing at Princeton University, a consortium of groups led by the Princeton Institute for Computational Science and Engineering (PICSciE) and Office of Information Technology's Research Computing. H.S. acknowledges support from the Swartz Program in Theoretical Neuroscience at Harvard, the Gatsby Charitable Foundation, and NIH grant NINDS (1U19NS104653).
[1] E. Tulving, in Organization of memory, edited by E. Tulving and W. Donaldson (Academic Press, 1972) pp. 381403.

[2] M. W. Howard and M. J. Kahana, Journal of Mathematical Psychology 46, 269 (2002).

[3] E. C. Tolman, Psychological Review 55, 189 (1948).

[4] J. R. Manns and H. Eichenbaum, Learning and Memory 16, 616 (2009).

[5] T. E. Behrens, T. H. Muller, J. C. Whittington, S. Mark, A. B. Baram, K. L. Stachenfeld, and Z. Kurth-Nelson, Neuron 100, 490 (2018).

[6] P. Smolensky, Artificial Intelligence 46, 159 (1990).

[7] T. A. Plate, (1994).

[8] C. Eliasmith, How to Build a Brain: A Neural Architecture for Biological Cognition (Oxford University Press, 2013).

[9] J. Feldman and D. Ballard, Cognitive Science 6, 205 (1982).

[10] K. J. Holyoak and J. E. Hummel, in Cognitive dynamics: Conceptual and representational change in humans and machines. (Lawrence Erlbaum Associates Publishers, Mahwah, NJ, US, 2000) pp. 229-263.

[11] P. Smolensky, "On the proper treatment of connectionism," in Philosophy, Mind, and Cognitive Inquiry: Resources for Understanding Mental Processes, edited by D. J. Cole, J. H. Fetzer, and T. L. Rankin (Springer Netherlands, Dordrecht, 1990) pp. 145-206.

[12] J. Feldman, Cognitive Neurodynamics 7, 1 (2013).

[13] R. Jackendorff, Foundations of language: Brain, meaning, grammar, evolution (Oxford University Press, 2002).

[14] K. Greff, S. van Steenkiste, and J. Schmidhuber, (2020), arXiv:2012.05208.

[15] O. Barak, M. Rigotti, and S. Fusi, Journal of Neuroscience 33, 3844 (2013).
[16] W. F. Podlaski, E. J. Agnes, and T. P. Vogels, bioRxiv , 2020.01.08.898528 (2020).

[17] P. Kanerva, in Artificial Neural Networks - ICANN 96, 1996 International Conference, Bochum, Germany, July 16-19, 1996, Proceedings (1996) pp. 869-873.

[18] S. D. Levy and R. Gayler, in Proceedings of the 2008 Conference on Artificial General Intelligence 2008: Proceedings of the First AGI Conference (IOS Press, NLD, 2008) p. $414-418$.

[19] D. A. Rachkovskij and E. M. Kussul, Neural Computation 13, 411 (2001), https://doi.org/10.1162/089976601300014592.

[20] D. Kleyko, D. A. Rachkovskij, E. Osipov, and A. Rahimi, , 1 (2021), arXiv:2112.15424.

[21] T. C. Stewart, T. Bekolay, and C. Eliasmith, Connection Science 23, 145 (2011).

[22] K. Schlegel, P. Neubert, and P. Protzel, Artificial Intelligence Review, 0123456789 (Springer Netherlands, 2021) arXiv:2001.11797.

[23] P. W. Battaglia, J. B. Hamrick, V. Bapst, A. SanchezGonzalez, V. Zambaldi, M. Malinowski, A. Tacchetti, D. Raposo, A. Santoro, R. Faulkner, C. Gulcehre, F. Song, A. Ballard, J. Gilmer, G. Dahl, A. Vaswani, K. Allen, C. Nash, V. Langston, C. Dyer, N. Heess, D. Wierstra, P. Kohli, M. Botvinick, O. Vinyals, Y. Li, and R. Pascanu, "Relational inductive biases, deep learning, and graph networks," (2018), arXiv:1806.01261 [cs.LG].

[24] A. Santoro, D. Raposo, D. G. Barrett, M. Malinowski, R. Pascanu, P. Battaglia, and T. Lillicrap, in Advances in Neural Information Processing Systems, Vol. 30, edited by I. Guyon, U. V. Luxburg, S. Bengio, H. Wallach, R. Fergus, S. Vishwanathan, and R. Garnett (Curran Associates, Inc., 2017). 
[25] M. Zaheer, S. Kottur, S. Ravanbakhsh, B. Poczos, R. R. Salakhutdinov, and A. J. Smola, in Advances in Neural Information Processing Systems 30, edited by I. Guyon, U. V. Luxburg, S. Bengio, H. Wallach, R. Fergus, S. Vishwanathan, and R. Garnett (Curran Associates, Inc., 2017) pp. 3391-3401.

[26] J. Devlin, M. W. Chang, K. Lee, and K. Toutanova, NAACL HLT 2019 - 2019 Conference of the North American Chapter of the Association for Computational Linguistics: Human Language Technologies - Proceedings of the Conference 1, 4171 (2019), arXiv:1810.04805.

[27] E. P. Frady, D. Kleyko, and F. T. Sommer, Neural Computation 30, 1449 (2018).

[28] J. C. Whittington, T. H. Muller, S. Mark, G. Chen, C. Barry, N. Burgess, and T. E. Behrens, Cell 183, 1249 (2020).

[29] J. C. R. Whittington, J. Warren, and T. E. J. Behrens, (2021), arXiv:2112.04035.

[30] D. J. Amit, H. Gutfreund, and H. Sompolinsky, Phys. Rev. Lett. 55, 1530 (1985).

[31] J. J. Hopfield, Proceedings of the National Academy of Sciences 79, 2554 (1982), https://www.pnas.org/content/79/8/2554.full.pdf.

[32] H. Sompolinsky and I. Kanter, Phys. Rev. Lett. 57, 2861 (1986).

[33] D. J. Amit, H. Gutfreund, and H. Sompolinsky, Phys. Rev. A 32, 1007 (1985).

[34] I. Kanter and H. Sompolinsky, Phys. Rev. A 35, 380 (1987).

[35] D. J. Amit, H. Gutfreund, and H. Sompolinsky, Annals of Physics 173, 30 (1987).

[36] A. Storkey, in Proceedings of the 7th International Conference on Artificial Neural Networks, ICANN '97 (Springer-Verlag, Berlin, Heidelberg, 1997) p. 451-456.

[37] A. Storkey and R. Valabregue, "A hopfield learning rule with high capacity storage of time-correlated patterns," (1997).

[38] A. Storkey and R. Valabregue, Neural Networks 12, 869 (1999).

[39] M. Nickel, L. Rosasco, and T. Poggio, in Proceedings of the Thirtieth AAAI Conference on Artificial Intelligence, AAAI'16 (AAAI Press, 2016) p. 1955-1961.

[40] M. V. Tsodyks and M. V. Feigel'man, Europhysics Letters (EPL) 6, 101 (1988).

[41] E. P. Frady, D. Kleyko, and F. T. Sommer, IEEE Transactions on Neural Networks and Learning Systems , 1 (2021).

[42] D. A. Rachkovskij, IEEE Transactions on Knowledge and Data Engineering 13, 261 (2001).

[43] D. A. Rachkovskij, E. M. Kussul, and T. N. Baidyk, Biologically Inspired Cognitive Architectures 3, 64 (2013).

[44] N. Hiratani and H. Sompolinsky, (to appear).

[45] R. A. Silver, Nature Reviews Neuroscience 11, 474 (2010).

[46] W. H. Mehaffey, B. Doiron, L. Maler, and R. W. Turner, Journal of Neuroscience 25, 9968 (2005).

[47] Y. Ahmadian, D. B. Rubin, and K. D. Miller, Neural Computation 25, 1994 (2013).

[48] P. Y. Deng and V. A. Klyachko, Communicative and Integrative Biology 4, 543 (2011).

[49] R. S. Zucker and W. G. Regehr, Annual Review of Physiology 64, 355 (2002).

[50] J. Ba, G. E. Hinton, V. Mnih, G. Deepmind, J. Z. Leibo, and C. Ionescu, , 1 (2016).
[51] I. Schlag, K. Irie, and J. Schmidhuber, (2021), arXiv:2102.11174.

[52] N. T. Franklin, K. A. Norman, C. Ranganath, J. M. Zacks, and S. J. Gershman, Psychological Review 127, 327 (2020).

[53] A. A. Kumar, Psychonomic Bulletin Review 28, 40 (2021).

[54] T. A. Plate, IEEE Transactions on Neural Networks 6, 623 (1995).

[55] A. J. Storkey, , 138 (1999).

[56] D. Kleinfeld and H. Sompolinsky, Biophysical Journal 54, 1039 (1988). 


\section{Appendix A: Holographic Reduced Representation}

\section{Overview}

[54] In holographic reduced representation (HRR) [7], the binding operation is given by the circular convolution

$$
g=\operatorname{cconv}(a, b)
$$

where the components of $g$ are given by

$$
g_{j}=\sum_{k=0}^{N-1} a_{k} b_{j-k}=\sum_{k=0}^{N-1} \sum_{m=0}^{N-1} \delta_{j, k+m} a_{k} b_{m}, j=0, \ldots, N-1
$$

and all subscripts are defined modulo $N$. For objects and attributes $a, b \in \mathbb{R}^{N}$, the binding operation creates a representation $g(a, b) \in \mathbb{R}^{N}$. The circular convolution operation is both associative and commutative, i.e. $\operatorname{cconv}(a, b)=$ $\operatorname{cconv}(b, a)$. The corresponding decoding operation is given by the circular correlation

$$
\phi=\operatorname{ccorr}(\widehat{S}, b)
$$

where components of $\phi$ are given by

$$
\phi_{j}=\sum_{k=0}^{N-1} \widehat{S}_{k} b_{j+k, \ell}=\sum_{k=0}^{N-1} \sum_{m=0}^{N-1} \delta_{j, m-k} \widehat{S}_{k} b_{m}, j=0, \ldots, N-1
$$

In general the circular correlation operation is neither associative nor commutative. However, it can be related to circular convolution by defining the involution of a vector $a$ as $a^{*}$ where $a_{j}^{*}=a_{-j}$. Then $\operatorname{ccorr}(a, b)=\operatorname{cconv}\left(a^{*}, b\right)$ and $a^{*}$ as serves as an approximate inverse to $a$ when both $a$ and $b$ are random vectors.

\section{Clean-up and SNR}

To quantify the effect of the clean-up operation described in the main text, we compute the statistics of the estimator $\hat{a}_{\ell}$ for the corresponding object $a_{\ell}$ decoded from a relational structure $\widehat{S}$ in the case that all and cues are random vectors with components drawn iid, i.e., $a_{i d} \sim \mathcal{N}\left(0, \frac{1}{N}\right), b_{i d} \sim \mathcal{N}\left(0, \frac{1}{N}\right)$ and decoding is done from a dictionary of size $D$.

Since the encoding operation is permutation invariant, the statistics for all $\hat{a}_{\ell}$ decoded from the full structure should be the same. For simplicity we set $\ell=1$ and consider $\hat{a}_{1}$. We index dictionary items so that indices $\ell<L$ correspond to the dictionary items contained in the structure $\widehat{S}$ and indices $L<\ell<D$ corresponds to dictionary items that are not contained in the structure. Note that

$$
\begin{aligned}
\left\langle a_{i, \ell} a_{j, \ell^{\prime}}\right\rangle & =\frac{1}{N} \delta_{i j} \delta_{\ell \ell^{\prime}} \\
\left\langle a_{i, \ell} a_{j, \ell^{\prime}} a_{k, s} a_{l, s^{\prime}}\right\rangle & =\frac{1}{N^{2}}\left(\delta_{i j} \delta_{\ell \ell^{\prime}} \delta_{k l} \delta_{s s^{\prime}}+\delta_{i k} \delta_{\ell s} \delta_{j l} \delta_{\ell^{\prime}} \delta_{s s^{\prime}}+\delta_{i l} \delta_{\ell s^{\prime}} \delta_{j k} \delta_{\ell^{\prime} s}\right)
\end{aligned}
$$

We start by expressing the estimator $\hat{a}_{1}$ as

$$
\hat{a}_{j, 1}=\sum_{k=0}^{N-1} \widehat{S}_{k} b_{j+k, 1}
$$

in terms of $a^{\prime}$ 's and $b$ 's, $\hat{a}_{j, 1}$ becomes

$$
\begin{aligned}
\hat{a}_{j, 1} & =a_{j, 1} \sum_{k=0}^{N-1}\left(b_{k, 1}\right)^{2}+\sum_{k=0, k^{\prime} \neq k}^{N-1} a_{k-k^{\prime}+j, 1} b_{k, 1} b_{k^{\prime}, 1}+\sum_{\ell=2}^{L} \sum_{k=0}^{N-1} \sum_{k^{\prime}=0}^{N-1} a_{k-k^{\prime}+j, \ell} b_{k, \ell} b_{k^{\prime}, 1} \\
& =(1+\epsilon) a_{j, 1}+\xi_{j, 1}+\xi_{j, 2}
\end{aligned}
$$


where we have defined the three noise terms as

$$
\begin{aligned}
\epsilon & =\sum_{k=0}^{N-1}\left(b_{k, 1}\right)^{2}-1 \\
\xi_{j, 1} & =\sum_{k=0}^{N-1} \sum_{k^{\prime}=0}^{N-1}\left(1-\delta_{k k^{\prime}}\right) b_{k, 1} b_{k^{\prime}, 1} a_{k-k^{\prime}+j, 1} \\
\xi_{j, 2} & =\sum_{\ell=2}^{L} \sum_{k=0}^{N-1} \sum_{k^{\prime}=0}^{N-1} b_{k, 1} b_{k^{\prime}, \ell} a_{k-k^{\prime}+j, \ell}
\end{aligned}
$$

Here, $\epsilon$ is noise due to inexact deviations from normalization of random Gaussian vectors from $1, \xi_{j, 1}$ is noise from interference elements of different elements of the cue within the same attribute $b_{1}$, and $\xi_{j, 2}$ and $\xi_{j, 2}$ are noise coming from interference between attribute $b_{1}$ with all other attributes $b_{\ell}$ contained in the structure. Since components of $a_{1}$ appear an odd number of times in each term of the sums in $\xi_{j, 1}$ and $\xi_{j, 2}$, we conclude $\left\langle\xi_{j, 1}\right\rangle=\left\langle\xi_{j, 2}\right\rangle=0$. Likewise, $\langle\epsilon\rangle=0$. Using Eqns. A5 and A6, we find that the second moments and correlation of the noise terms are given by

$$
\begin{aligned}
& \left\langle\epsilon^{2}\right\rangle=1+\left(\sum_{k=0}^{N-1} \sum_{q=0}^{N-1}\left\langle b_{k}^{2} b_{q}^{2}\right\rangle\right)-2 \sum_{k=0}^{N-1}\left\langle b_{k}^{2}\right\rangle \\
& =\sum_{k=0}^{N-1} \sum_{q=0}^{N-1} \frac{1}{N^{2}}\left(1+2 \delta_{k q}\right)-1 \\
& =\frac{2}{N} \\
& \left\langle\xi_{j, 1}^{2}\right\rangle=\sum_{k=0}^{N-1} \sum_{k^{\prime}=0}^{N-1} \sum_{q=0}^{N-1} \sum_{q^{\prime}=0}^{N-1}\left(1-\delta_{k k^{\prime}}\right)\left(1-\delta_{q q^{\prime}}\right)\left\langle b_{k, 1} b_{k^{\prime}, 1} b_{q, 1} b_{q^{\prime}, 1}\right\rangle\left\langle a_{k-k^{\prime}+j, 1} a_{q-q^{\prime}+j, 1}\right\rangle \\
& =\sum_{k=0}^{N-1} \sum_{k^{\prime}=0}^{N-1} \sum_{q=0}^{N-1} \sum_{q^{\prime}=0}^{N-1} \frac{1}{N^{3}}\left(\delta_{k q} \delta_{k^{\prime} q^{\prime}}-\delta_{k k^{\prime}} \delta_{q q^{\prime}} \delta_{k q}\right) \\
& =\frac{(N-1)}{N^{2}} \\
& \left\langle\xi_{j, 2}^{2}\right\rangle=\sum_{\ell=2}^{L} \sum_{\ell^{\prime}=2}^{L} \sum_{k=0}^{N-1} \sum_{k^{\prime}=0}^{N-1} \sum_{q=0}^{N-1} \sum_{q^{\prime}=0}^{N-1}\left\langle b_{k, 1} b_{k^{\prime}, \ell} b_{q, 1} b_{q^{\prime}, \ell^{\prime}}\right\rangle\left\langle a_{k-k^{\prime}+j, \ell} a_{q-q^{\prime}+j, \ell^{\prime}}\right\rangle \\
& =\sum_{\ell=2}^{L} \sum_{\ell^{\prime}=2}^{L} \sum_{k=0}^{N-1} \sum_{k^{\prime}=0}^{N-1} \sum_{q=0}^{N-1} \sum_{q^{\prime}=0}^{N-1} \frac{1}{N^{3}}\left(\delta_{1 \ell} \delta_{\ell \ell^{\prime}} \delta_{k k^{\prime}} \delta_{q q^{\prime}}+\delta_{\ell \ell^{\prime}} \delta_{k q} \delta_{k^{\prime} q^{\prime}}+\delta_{1 \ell} \delta_{\ell \ell^{\prime}} \delta_{k q^{\prime}} \delta_{k^{\prime} q} \delta_{k-k^{\prime}, q-q^{\prime}}\right) \\
& =\frac{L}{N}+\frac{1}{N^{2}} \\
& \left\langle\xi_{j, 1} \xi_{j, 2}\right\rangle=\sum_{\ell=2}^{L} \sum_{k=0}^{N-1} \sum_{k^{\prime}=0}^{N-1} \sum_{q=0}^{N-1} \sum_{q^{\prime}=0}^{N-1}\left(1-\delta_{k k^{\prime}}\right)\left\langle b_{k, 1} b_{k^{\prime}, 1} b_{q, 1} b_{q^{\prime}, \ell}\right\rangle\left\langle a_{k-k^{\prime}+j, 1} a_{q-q^{\prime}+j, \ell}\right\rangle \\
& =0
\end{aligned}
$$

From Eqn. A8, we see the noise in each component of the estimator is

$$
\frac{\left\langle\delta \hat{a}_{j, \ell}\right\rangle}{\left\langle a_{j, \ell}^{2}\right\rangle} \sim L
$$

We can compare the SNR for the estimator $\hat{a}_{1}$ with the SNR for the average overlap of the estimator with items in the dictionary $a_{d} \cdot\left\langle\hat{a}_{1}\right\rangle$. We start by considering statistics of the overlap of the estimator with the correct pattern $a_{d} \cdot \hat{a}_{1}$. This can be expressed as

$$
a_{d} \cdot \hat{a}_{1}=(1+\epsilon) a_{d} \cdot a_{1}+a_{d} \cdot\left(\xi_{1}+\xi_{2}\right)
$$


We see from Eqn. A17 that $\left\langle a_{1} \cdot \hat{a}_{1}\right\rangle=1$ and $\left\langle a_{d} \cdot \hat{a}_{1}\right\rangle=0$, so the estimator is unbiased. The second moment is given by

$$
\begin{aligned}
\left\langle\left(a_{d} \cdot \hat{a}_{1}\right)^{2}\right\rangle & =\left\langle\sum_{j=0}^{N-1} \sum_{j^{\prime}=0}^{N-1} \sum_{k=0}^{N-1} \sum_{k^{\prime}=0}^{N-1} \sum_{q=0}^{N-1} \sum_{q^{\prime}=0}^{N-1} \sum_{\ell=1}^{L} \sum_{\ell^{\prime}=1}^{L} b_{k, 1} b_{k^{\prime}, \ell} a_{k-k^{\prime}+j, \ell} a_{j, d} b_{q, 1} b_{q^{\prime}, \ell^{\prime}} a_{q-q^{\prime}+j^{\prime}, \ell^{\prime}} a_{j^{\prime}, d}\right\rangle \\
& =\sum_{j=0}^{N-1} \sum_{j^{\prime}=0}^{N-1} \sum_{k=0}^{N-1} \sum_{k^{\prime}=0}^{N-1} \sum_{q=0}^{N-1} \sum_{q^{\prime}=0}^{N-1} \sum_{\ell=1}^{L} \sum_{\ell^{\prime}=1}^{L}\left\langle b_{k, 1} b_{k^{\prime}, \ell} b_{q, 1} b_{q^{\prime}, \ell^{\prime}}\right\rangle\left\langle a_{k-k^{\prime}+j, \ell} a_{j, d} a_{q-q^{\prime}+j^{\prime}, \ell^{\prime}} a_{j^{\prime}, d}\right\rangle \\
& =\sum_{j=0}^{N-1} \sum_{j^{\prime}=0}^{N-1} \sum_{k=0}^{N-1} \sum_{k^{\prime}=0}^{N-1} \sum_{q=0}^{N-1} \sum_{q^{\prime}=0}^{N-1} \sum_{\ell=1}^{L} \sum_{\ell^{\prime}=1}^{L} \frac{1}{N^{4}}\left(\delta_{k k^{\prime}} \delta_{q q^{\prime}} \delta_{d \ell} \delta_{1 \ell^{\prime}} \delta_{\ell \ell^{\prime}}+\delta_{k k^{\prime}} \delta_{q q^{\prime}} \delta_{k q} \delta_{\ell \ell^{\prime}} \delta_{1 \ell}+\delta_{k k^{\prime}} \delta_{q q^{\prime}} \delta_{j j^{\prime}} \delta_{d \ell} \delta_{1 \ell^{\prime}} \delta_{\ell \ell^{\prime}}\right. \\
& +\delta_{k k^{\prime}} \delta_{q q^{\prime}} \delta_{k q} \delta_{\ell \ell^{\prime}} \delta_{\ell d}+\delta_{k q} \delta_{k^{\prime} q^{\prime}} \delta_{j j^{\prime}} \delta_{\ell \ell^{\prime}}+\delta_{k q} \delta_{k^{\prime} q^{\prime}} \delta_{j j^{\prime}} \delta_{\ell \ell^{\prime}} \delta_{\ell d}+\delta_{k k^{\prime}} \delta_{q q^{\prime}} \delta_{k q} \delta_{1 \ell^{\prime}} \delta_{d \ell} \delta_{\ell \ell^{\prime}}+\delta_{k k^{\prime}} \delta_{q q^{\prime}} \delta_{k q} \delta_{j j^{\prime}} \delta_{\ell \ell^{\prime}} \delta_{1 \ell} \\
& \left.+\delta_{k k^{\prime}} \delta_{q q^{\prime}} \delta_{k q} \delta_{j j^{\prime}} \delta_{1 \ell} \delta_{d \ell^{\prime}} \delta_{\ell \ell^{\prime}}\right) \\
& \left.=\delta_{1 d}+\frac{L}{N}+\frac{1}{N}\left(1+2 \delta_{1 d}+2 \Theta(L-d)\right)+\frac{1}{N^{2}}\left(1+\delta_{1 d}\right)\right)
\end{aligned}
$$

To summarize

$$
\begin{aligned}
&\left\langle a_{d} \cdot \hat{a}_{1}\right\rangle= \begin{cases}1 & d=1 \\
0 & 1<d \leq L \\
0 & L<d\end{cases} \\
& \Sigma_{1 d}^{2}=\left\langle\left(a_{d} \cdot \hat{a}_{1}-\left\langle a_{d} \cdot \hat{a}_{1}\right\rangle\right)^{2}\right\rangle= \begin{cases}\frac{L+5}{N}+\frac{2}{N^{2}} & d=1 \\
\frac{L+3}{N}+\frac{1}{N^{2}} & 1<d \\
\frac{L+1}{N}+\frac{1}{N^{2}} & L<d\end{cases}
\end{aligned}
$$

For $L \ll N$ SNR for overlaps defined in Eqn. 7 of the main text is then approximately

$$
\frac{\left\langle a_{1} \cdot \hat{a}_{1}\right\rangle^{2}}{\left\langle\left(a_{d} \cdot \hat{a}_{1}\right)^{2}\right\rangle} \approx \frac{N}{L}
$$

\section{Binarization and Empirical SNR}

When decoding from a binarized structure $\sigma=\operatorname{sgn}(\widehat{S})$, the estimator $\hat{a}_{d}$ is given by

$$
\hat{a}_{j d}=\sum_{k=0}^{N-1} \sigma_{k} b_{k+j, d}
$$

where

$$
\sigma_{j}=\operatorname{sgn}\left(\sum_{k=0}^{N-1} \sum_{\ell=1}^{L} a_{k, \ell} b_{j-k, \ell}\right)
$$

To compute the SNR, we start by evaluating the overlap between the estimator $\hat{a}_{1}$ with items in the dictionary $\left\langle a_{d} \cdot \hat{a}_{1}\right\rangle$ which can be expressed as

$$
\left\langle a_{d} \cdot \hat{a}_{1}\right\rangle=\left\langle\sum_{j=0}^{N-1} \sum_{k=0}^{N-1} a_{j, d} b_{k, 1} \operatorname{sgn}\left(a_{j, 1} b_{k, 1}+\sum_{q \neq k}^{N-1} a_{k-q+j, 1} b_{q, 1}+\sum_{\ell=2}^{L} \sum_{q=0}^{N-1} a_{k-q+j, \ell} b_{q, \ell}\right)\right\rangle
$$

While individual products of elements $a_{\ell, i} b_{\ell, j}$ are not Gaussian distributed, the expectation $\left\langle a_{d} \cdot \hat{a}_{1}\right\rangle$ is the sum of $N^{2}$ random variables with correlations only occurring at higher order as the expectation of the product of different elements of each circular convolution is zero. Since all terms contained in the sums over indiced $j$ and $k$ in Eqn. A24 
are only correlated at higher order, we can approximate each term in the sum as independent which gives us

$$
\left\langle a_{d} \cdot \hat{a}_{1}\right\rangle \approx N^{2}\left\langle a_{0, d} b_{0,1}\left(\operatorname{sgn}\left(a_{0,1} b_{0,1}+\sum_{q=1}^{N-1} a_{N-q, 1} b_{q, 1}+\sum_{\ell=2}^{L} \sum_{q=0}^{N-1} a_{N-q, \ell} b_{q, \ell}\right)\right)\right\rangle
$$

We now define the following three random variables

$$
\begin{aligned}
z_{1} & =a_{0,1} b_{0,1} \\
z_{d} & =a_{0, d} b_{0,1} \\
\xi & =\sum_{q=1}^{N-1} a_{N-q, 1} b_{q, 1}+\sum_{\ell=2}^{L} \sum_{q=0}^{N-1} a_{N-q, \ell} b_{q, \ell}
\end{aligned}
$$

where $x_{1}$ is a signal term and $\xi$ is a noise term within the sign function. $\left\langle z_{1}\right\rangle=\left\langle z_{2}\right\rangle=\langle\xi\rangle=0$ and the variances are given by

$$
\begin{aligned}
\Sigma_{z_{1}}^{2} & =\frac{1}{N^{2}} \\
\Sigma_{z_{\ell}}^{2} & =\frac{1}{N^{2}} \\
\Sigma_{\xi}^{2} & =\frac{(N-1)}{N^{2}}+\frac{L}{N} \sim \frac{L}{N}
\end{aligned}
$$

For $d=1, z_{1}=z_{\ell}=z$ and $L \gg 1$ we can treat $z$ and $\xi$ as Gaussian. In this approximation $\left\langle a_{1} \cdot \hat{a}_{1}\right\rangle$ becomes

$$
\begin{aligned}
\left\langle a_{1} \cdot \hat{a}_{1}\right\rangle & \approx N^{2} \int_{-\infty}^{\infty} \frac{d z}{\sqrt{2 \pi \Sigma_{z}^{2}}} \int_{-\infty}^{\infty} \frac{d \xi}{\sqrt{2 \pi \Sigma_{\xi}^{2}}} e^{-\frac{z^{2}}{2 \Sigma_{z}^{2}}-\frac{\xi^{2}}{2 \Sigma_{\xi}^{2}}} z \operatorname{sgn}(z+\xi) \\
& =\sqrt{\frac{2}{\pi}} \frac{N^{2} \Sigma_{z}^{2}}{\sqrt{\Sigma_{z}^{2}+\Sigma_{\xi}^{2}}} \\
& \sim \sqrt{\frac{2 N}{\pi L}}
\end{aligned}
$$

Likewise, the second moment is given by

$$
\left\langle\left(a_{1} \cdot \hat{a}_{1}\right)^{2}\right\rangle=\left\langle\left(a_{d} \cdot \hat{a}_{1}\right)^{2}\right\rangle \approx N^{2} \int_{-\infty}^{\infty} \frac{d z}{\sqrt{2 \pi \Sigma_{z}^{2}}} e^{-\frac{z^{2}}{2 \Sigma}} \int_{-\infty}^{\infty} \frac{d \xi}{\sqrt{2 \pi \Sigma_{\xi}^{2}}} e^{-\frac{\xi^{2}}{2 \Sigma_{\xi}^{2}}} z^{2}=1
$$

Putting Eqns. A32 and A33 together, the SNR of overlap when decoding $a_{1}$ from the binarized structure $\sigma$ is given by

$$
\mathrm{SNR}=\frac{\left\langle a_{1} \cdot \hat{a}_{1}\right\rangle^{2}}{\left\langle\left(a_{d} \cdot \hat{a}_{1}\right)^{2}\right\rangle} \sim \frac{2}{\pi} \frac{N}{L}
$$

We see from Eqn. A21 that binarizing $\widehat{S}$ has decreased the SNR by a constant factor of $\frac{2}{\pi}$.

Finally, we consider decoding from a degraded binarized structure which is a Hamming distance $\frac{m-1}{2}$ from the original structure. In this case, the effective SNR is found by making the replacement $\left\langle a_{1} \cdot \hat{a}_{1}\right\rangle \rightarrow m$ in Eqn. A34. From this, we see that $\operatorname{SNR}(m)$ should take the form

$$
\operatorname{SNR}(m)=\frac{2}{\pi}\left(\frac{m}{\Sigma}\right)^{2} \sim \frac{2 m^{2}}{\pi} \frac{N}{L}
$$

When $\hat{a}_{1}$ is decoded from an imperfectly retrieved structured from memory, we can instead make the replacement $\left\langle a_{1} \cdot \hat{a}_{1}\right\rangle \rightarrow \sqrt{c} m$ where $c \sim O(1)$ is a constant factor accounting for differences in overlap of the structure with 
relations within and outside the retrieval cue. Then $\operatorname{SNR}(m)$ takes the modified form

$$
\operatorname{SNR}(m) \sim \frac{2 c m^{2}}{\pi} \frac{N}{L}
$$

then averaging Eqn. 6 from the main text over the distribution $p(m)$, the decoding error is closely approximated by

$$
P_{\epsilon} \approx \int d m p(m) \int_{-\infty}^{\infty} D z\left(1-H(-z-\sqrt{\operatorname{SNR}(m)})^{D}\right)
$$

\section{Memory Initialization}

We now determine the average initial overlap $m_{0}$ between a binarized retrieval structure $\sigma_{0}$ containing $L_{0}$ of the $L$ relations in the binarized full structure $\sigma$ in the case of random $a_{\ell}$ 's and $b_{\ell}$ 's are random vectors with components drawn iid as before. An expression for the average overlap $m_{0}$ between $\sigma_{0}$ and $\sigma$ can be written as

$$
m_{0}=\left\langle\frac{1}{N} \sum_{j=0}^{N-1} \operatorname{sgn}\left(\widehat{S}_{j}\right) \operatorname{sgn}\left(\widehat{S}_{j, 0}\right)\right\rangle
$$

The overlap between components of the unbinarized structures $\widehat{S}_{j}$ and $\widehat{S}_{j^{\prime} 0}$ with HRR binding is given by

$$
\begin{aligned}
\left\langle\widehat{S}_{j} \widehat{S}_{j^{\prime} 0}\right\rangle & \left.=\sum_{\ell=1}^{L} \sum_{\ell^{\prime}=1}^{L_{0}} \sum_{k=0}^{N-1} \sum_{k^{\prime}=0}^{N-1} \sum_{p=0}^{N-1} \sum_{p^{\prime}=0}^{N-1} \delta_{p^{\prime}, j^{\prime}-k^{\prime}} \delta_{p, j-k} a_{k, \ell} a_{k^{\prime}, \ell^{\prime}} b_{p, \ell} b_{p^{\prime}, \ell^{\prime}}\right\rangle \\
& =\left\langle\sum_{\ell=1}^{L_{0}} \sum_{k=0}^{N-1} \delta_{j j^{\prime}} a_{k, \ell}^{2} b_{j-k, \ell}^{2}\right\rangle \\
& =\frac{L_{0}}{N} \delta_{j j^{\prime}}
\end{aligned}
$$

For large $N$ we can approximate the sum in Eqn. A38 by treating the individual terms as as independent since there are no lower order correlations between terms. We can then define the random variables $z_{1}=\widehat{S}_{j 0}$ and $z_{2}=\widehat{S}_{j}-\widehat{S}_{j 0}$ as approximately Gaussian distributed with zero mean and variances $\Sigma_{z_{1}}^{2}=\frac{L_{0}}{N}$ and $\Sigma_{z_{2}}^{2}=\frac{\left(L-L_{0}\right)}{N}$ respectively. Then, Eqn. A38 can be approximated as

$$
\begin{aligned}
m_{0} & \approx \int_{-\infty}^{\infty} \frac{d z_{1}}{\sqrt{\sum_{z_{1}}^{2}}} e^{-\frac{z_{1}^{2}}{2 \Sigma_{z_{1}}^{2}}} \int_{-\infty}^{\infty} \frac{d z_{2}}{\sqrt{2 \pi \Sigma_{z_{2}}^{2}}} e^{-\frac{z_{2}^{2}}{2 \Sigma_{z_{2}}^{2}} \operatorname{sgn}\left(z_{1}\right) \operatorname{sgn}\left(z_{1}+z_{2}\right)} \\
& \approx \frac{2}{\pi} \arctan \left(\frac{\Sigma_{z_{1}}}{\Sigma_{z_{2}}}\right) \\
& \approx \frac{2}{\pi} \arctan \left(\left(\frac{\frac{L_{0}}{L}}{1-\frac{L_{0}}{L}}\right)^{\frac{1}{2}}\right)
\end{aligned}
$$

For $L_{0} \ll L$ Eqn. A40 can be further approximated as

$$
m_{0} \approx \frac{2}{\pi} \sqrt{\frac{L_{0}}{L}}
$$

This implies that creating retrieval structures with $L_{0}$ out of $L$ relations will result in retrieval cues with average overlap $m_{0}$ with the full memorized structure. The variance of the distribution of initial overlaps will vanish as $N \rightarrow \infty$. 


\section{Appendix B: Decoding Error}

For ML decoding with a dictionary, we derive a good approximation for the decoding error in terms of the size of the decoding dictionary $D$ and the mean and variance of the overlap between the estimator with items in the dictionary. We define the overlap $\mathcal{O}_{d 1}$ between a dictionary item $a_{d}$ with the estimator $\hat{a}_{1}$ for $a_{1}$ decoded from a structure $\widehat{S}$ of length $L$ as

$$
\mathcal{O}_{1 d}=\hat{a}_{1} \cdot a_{d}
$$

here we index items so that $d \geq L$ corresponds to overlap with patterns within $\widehat{S}$ and $L<d$ corresponds to overlaps with other items in the dictionary. For MLE decoding, an error occurs if $\max \left(\mathcal{O}_{12}, \ldots, \mathcal{O}_{1 D}\right)>\mathcal{O}_{11}$. The probability of error $P_{\epsilon}$ is given by

$$
P_{\epsilon}=1-\int_{-\infty}^{\infty} d \mathcal{O}_{11} P\left(\mathcal{O}_{11}\right)\left(\int_{-\infty}^{\mathcal{O}_{11}} d \mathcal{O}_{1 L} P\left(\mathcal{O}_{1 L}\right)\right)^{L-1}\left(\int_{-\infty}^{\mathcal{O}_{11}} d \mathcal{O}_{1 D} P\left(\mathcal{O}_{1 D}\right)\right)^{D-L}
$$

For large $N$, each $\mathcal{O}_{1 d}$ essentially behaves as an independent Gaussian random variable with mean $\mu_{1 d}$ and variance $\Sigma_{1 d}$. For unbiased decoding schemes, $\hat{a}_{1}^{\mu}, \mu_{1 L}=\mu_{1 D}=0$. In general, we can consider decoding from a noisy or corrupted structured by setting $\mu_{11}=\mu$, where $\mu$ is related to the overlap of the noisy structure with the correct structure. Then $P_{\epsilon}$ can be very well approximated as

$$
P_{\epsilon} \approx \int_{-\infty}^{\infty} D z\left(1-H\left(-\frac{\Sigma_{11} z+\mu}{\Sigma_{1 L}}\right)^{L-1} H\left(-\frac{\Sigma_{11} z+\mu}{\Sigma_{1 D}}\right)^{D-L}\right)
$$

where $D z=\frac{d z}{\sqrt{2 \pi}} e^{-\frac{z^{2}}{2}}$.

For $\operatorname{HRR} \Sigma_{11} \approx \Sigma_{1 L} \approx \Sigma_{1 D}$ to leading order in $\frac{1}{N}$. Defining $\Sigma$ to be the leading order term in the variance, we obtain

$$
P_{\epsilon} \approx \int_{-\infty}^{\infty} D z\left(1-H\left(-z-\frac{\mu}{\Sigma}\right)^{D}\right)
$$

Identifying $\frac{\mu^{2}}{\Sigma^{2}}$ with the SNR of overlaps, Eqn. B4 becomes

$$
P_{\epsilon} \approx \int_{-\infty}^{\infty} D z\left(1-H(-z-\sqrt{\mathrm{SNR}})^{D}\right)
$$

given in the main text. Making the identification $\mu=\sqrt{c} m$, the decoding error after retrieval from memory for HRR is given by

$$
\begin{aligned}
P_{\epsilon} & \approx \int d m p(m) \int_{-\infty}^{\infty} D z\left(1-H(-z-\sqrt{\mathrm{SNR}(m)})^{D}\right) \\
& \approx \int d m p(m) \int_{-\infty}^{\infty} D z\left(1-H\left(-z-\sqrt{\frac{2 c m^{2}}{\pi} \frac{N}{L}}\right)^{D}\right)
\end{aligned}
$$

where $p(m)$ takes the same form as in the main text. For $N \rightarrow \infty$

$$
P_{\epsilon} \approx \int_{-\infty}^{\infty} D z\left(1-H\left(-z-\sqrt{\frac{2 c\langle m\rangle^{2}}{\pi}} \frac{N}{L}\right)^{D}\right)
$$

We can understand the dependence of $P_{\epsilon}$ on the SNR and D in the large SNR regime by obtaining a saddlepoint approximation for the expression for $P_{\epsilon}$ given in Eqn. B4. We start by rewriting Eqn. B4 as

$$
P_{\epsilon} \approx \int_{-\infty}^{\infty} \frac{d z}{\sqrt{2 \pi}} e^{f(z)}
$$


where $f(z)$ is given by

$$
f(z)=-\frac{z^{2}}{2}+\log \left(1-H\left(-z-\frac{\mu}{\Sigma}\right)^{D}\right)
$$

From Eqn. B4, we see $P_{\epsilon} \ll 1$ for $H\left(z+\frac{\mu}{\Sigma}\right) \ll 1$ as $H\left(-z-\frac{\mu}{\Sigma}\right)=1-H\left(z+\frac{\mu}{\Sigma}\right)$. So we are interested in the regime where $1 \ll z+\frac{\mu}{\Sigma}$. In this regime, $f(z)$ is very large, so the integral in $P_{\epsilon}$ can be approximated its saddlepoint value

$$
P_{\epsilon} \approx \frac{1}{\sqrt{\left|f^{\prime \prime}\left(z_{0}\right)\right|}} e^{f\left(z_{0}\right)}
$$

where $z_{0}$ is given by the solution of

$$
z=-\frac{D H\left(-z-\frac{\mu}{\Sigma}\right)^{D-1}}{1-H\left(-z-\frac{\mu}{\Sigma}\right)^{D}} \frac{e^{-\frac{\left(z+\frac{\mu}{\nu}\right)^{2}}{2}}}{\sqrt{2 \pi}}
$$

In this regime we can make the approximation

$$
H\left(-z-\frac{\mu}{\Sigma}\right)^{-D} \approx e^{D H\left(z+\frac{\mu}{\Sigma}\right)}
$$

which allows us to approximate Eqn. B11 as

$$
\begin{aligned}
z & =-\frac{D H\left(-z-\frac{\mu}{\Sigma}\right)^{D-1}}{1-H\left(-z-\frac{\mu}{\Sigma}\right)^{D}} \frac{e^{-\frac{\left(z+\frac{\mu}{\Sigma}\right)^{2}}{2}}}{\sqrt{2 \pi}} \\
& \approx-\frac{D H\left(z+\frac{\mu}{\Sigma}\right)}{H\left(-z-\frac{\mu}{\Sigma}\right)^{-D}-1}\left(z+\frac{\mu}{\Sigma}\right) \\
& \approx-\frac{D H\left(z+\frac{\mu}{\Sigma}\right)}{e^{D H\left(z+\frac{\mu}{\Sigma}\right)}-1}\left(z+\frac{\mu}{\Sigma}\right)
\end{aligned}
$$

where for $z+\frac{\mu}{\Sigma} \gg 1$ we used

$$
H\left(z+\frac{\mu}{\Sigma}\right) \approx \frac{e^{-\frac{x^{2}}{2 \Sigma^{2}}}}{\sqrt{2 \pi}\left(z+\frac{\mu}{\Sigma}\right)}
$$

From Eqn. B13 we see that for $D H\left(\frac{x}{\Sigma}\right) \ll 1, z \rightarrow-\frac{\mu}{2 \Sigma}$ and $f^{\prime \prime}\left(-\frac{\mu}{2 \Sigma}\right) \approx-1$. Identifying SNR $=\frac{\mu^{2}}{\Sigma^{2}}$ with the $S N R$ of overlaps, the saddlepoint approximation for the error is

$$
\begin{aligned}
P_{\epsilon} & \approx \int_{-\infty}^{\infty} D z\left(1-H(-z-\sqrt{\mathrm{SNR}})^{D}\right) \\
& \approx \int_{-\infty}^{\infty} D z e^{\log \left(1-e^{-D H(z+\sqrt{\mathrm{SNR}})}\right)} \\
& \approx e^{-\frac{\mathrm{SNR}}{8}}\left(1-e^{-\sqrt{\frac{2}{\pi \mathrm{SNR}}} D e^{-\frac{\mathrm{SNR}}{8}}}\right)
\end{aligned}
$$

This can be further approximated as

$$
P_{\epsilon} \approx \sqrt{\frac{2}{\pi \mathrm{SNR}}} D e^{-\frac{\mathrm{SNR}}{4}}
$$

We can insert the SNR of overlaps into Eqn. B17 to determine the limits on the size of $D$ for good decoding. 


\section{Appendix C: Insights from Random Patterns}

\section{Network Order Parameters}

We can characterize the quality of memory retrieval on average by formally defining three network order parameters which quantify the overlap of the network state with the stored memories at each time step. The first is

$$
m^{\mu}(t)=\frac{1}{N} \sum_{i=1}^{N} \sigma_{i}^{\mu} \sigma_{i}(t)
$$

which represents the overlap of the network state $\sigma(t)$ with each pattern $\mu$ at time $t$. If the initial state of the network has an $O(1)$ with a small number of patterns, i.e., $m_{0}=m^{\mu}(0)$, the memory retrieval process can be sufficiently described by including an additional order parameter

$$
r^{\mu}(t)=\frac{1}{\alpha} \sum_{\nu \neq \mu}^{P} m^{\nu}(t)^{2}
$$

which represents the overlap of the state with all patterns except for $\mu$. We can also define

$$
m^{\mu 0}(t)=\frac{1}{N} \sum_{i=1}^{N} \sigma_{i}^{\mu}(0) \sigma_{i}(t)
$$

as the overlap of the state at time $t$ with the initial state of the network after attempting to retrieve the $\mu$ th pattern. While $m^{0}$ is typically not considered for random patterns, it becomes relevant in the case of structured knowledge, where the retrieval cue can be constructed from a subset of relations rather than simply a corrupted version of a memory.

For large $N$ the distributions of order parameters over fixed retrieval conditions, i.e., $p(m), p(r)$ and $p\left(m^{0}\right)$, are sharply peaked at the retrieval averaged values, denoted by $\langle m\rangle,\langle r\rangle$, and $\left\langle m^{0}\right\rangle$. In the limit $N \rightarrow \infty$, these quantities depend solely on $m_{0}$ and $\alpha$.

\section{Retrieval Dynamics}

When retrieving structures from memory, in the absence of noise, the update equation for the state of a neuron at time $t$ is given by

$$
\sigma_{i}(t)=\operatorname{sgn}\left(\sum_{j} J_{i j} \sigma_{j}(t-1)\right)
$$

For all of the simulations in the main text, we consider parallel updates where all of the neurons $1 \leq i \leq N$. We find that serial updates give qualitatively similar results to parallel updates, where in general for large $N$

$$
\langle m\rangle \sim f(\alpha) m_{0}
$$

(which is equivalent to the value of $f(\alpha)$ defined for $\bar{m}$ in the main text for $N \rightarrow \infty$ ). We find that serial updates obey the form in Eqn. C5 with a slightly smaller value of the coefficient $f(\alpha)$. Additionally, for serial dynamics, we can consider the robustness of our results under addition of noise in the updates. To do this, we use the Metropolis algorithm to obtain the final equilibrium state, where the amplitude of the noise is controlled by the inverse temperature $\beta$. At each update, the acceptance probability is given by

$$
p\left(\sigma_{i} \rightarrow-\sigma_{i}\right)=\min \left(1, e^{-2 \beta h_{i} \sigma_{i}}\right)
$$

We see that Eqn. C6 becomes equivalent to Eqn. C4 for $\beta=\infty$, i.e., the noiseless case. In Fig. 10 we show the average overlap $\langle m\rangle$ of the network state with the cued memory as a function of time for several different initial overlaps $m_{0}$ retrieved through parallel dynamics and serial dynamics for several different values of $\beta$. In Fig. 11 we show $\langle m\rangle$, 

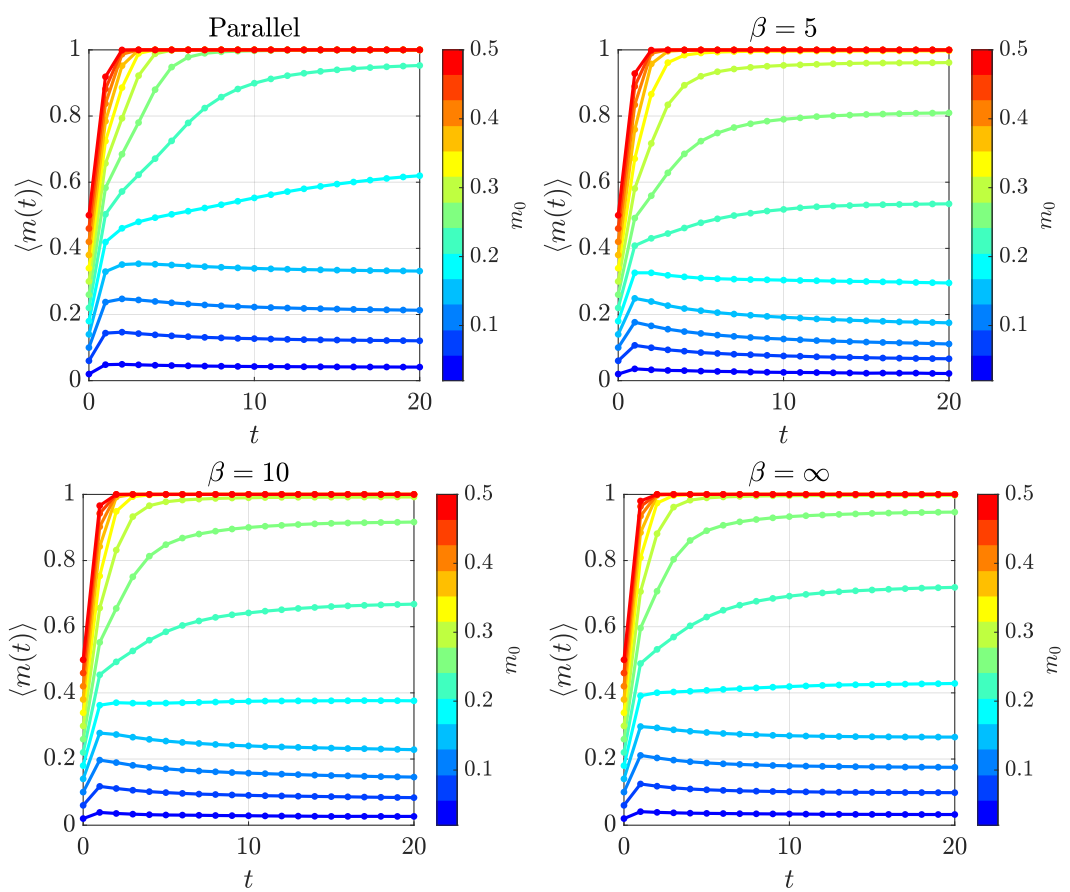

FIG. 10. Evolution of the averaged overlap order parameter $\langle m\rangle$ over $T=20$ updates compared for parallel updates serial dynamics at zero noise $(\beta=\infty)$, and serial dynamics with noise amplitude determined by inverse temperature $\beta=5,10$, for several different initial conditions $m_{0}$. The pseudo-inverse rule is used to store the memories. $\alpha=0.1, N=1000$, and 50 trials are used for all figures.
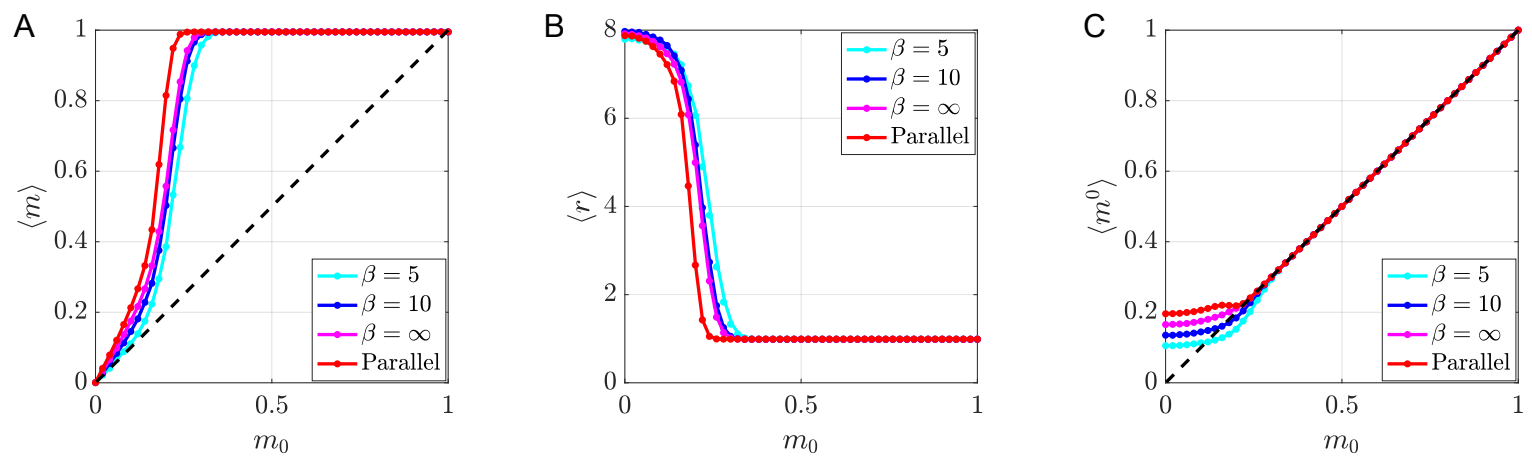

FIG. 11. Comparison of averaged order parameters $\langle m\rangle(\mathrm{A}),\langle r\rangle(\mathrm{B})$, and $\left\langle m^{0}\right\rangle(\mathrm{C})$, after $T=20$ updates as functions of retrieval cue overlap $m_{0}$ are compared for parallel updates and serial updates for various values of $\beta$. The dashed line in $\mathrm{A}$ and $\mathrm{C}$ is shown to compare the value of the final overlap with the cued memory $\langle m\rangle$ and the initial state $\left\langle m^{0}\right\rangle$ to the initial overlap with the cued memory. The pseudo-inverse rule is used to store memories and $\alpha=0.1, N=1000,50$ trials are used for all figures.

$\langle r\rangle,\left\langle m^{0}\right\rangle$ as function of retrieval cue overlap $m_{0}$. We see that the highest overlap is achieved for parallel updates when the serial updates are done in random order (alternative orders are discussed in [34]). However, the results are qualitatively similar for the different dynamics shown, demonstrating that small amounts of noise have little effect.

In Fig. $11 \mathrm{~A}$ and $\mathrm{C}$, we see that outside of the basin of attraction of the cued memory, i.e., values of $m_{0}$ where $\langle m\rangle<1$, the final network state retains some memory of the initial state reflected in $\left\langle m^{0}\right\rangle>m_{0}$

\section{Comparing Learning Rules}

The storage and retrieval of random memories in Hopfield networks near saturation, i.e., when the number of memories $P$ scaling linearly in $N$, is limited by interference between different memories [30]. The overlaps between 
different memories are characterized by the following matrix

$$
C^{\mu \nu}=\frac{1}{N} \sum_{i=1}^{N} \sigma_{i}^{\mu} \sigma_{i}^{\nu}
$$

Note that $C^{\mu \nu}$ is a $P \times P$ matrix that has the form of a sample covariance matrix.

We can examine the interference between memories by looking at the resulting noise term in the local field, which is given

$$
h_{i}(t)=\sum_{j} J_{i j} \sigma_{j}(t)
$$

From Eqn. C4, we see that a memory is a fixed point if $\sigma_{i}^{\mu} h_{i}>0$ for all $i=1, \ldots, N$. The elimination of the self-coupling term $J_{i i}$ greatly increases the basins of attraction of the pattern. In the limit $N, P \rightarrow \infty$ this can be accomplished by replacing $J_{i j}$ with $J_{i j}-\alpha \delta_{i j}[34]$.

We start by considering the Hebb rule, with synaptic weight matrix given by

$$
J_{i j}^{H}=\sum_{\mu}^{P} \sigma_{i}^{\mu} \sigma_{j}^{\mu}, i \neq j
$$

The local field for the Hebb rule can be expressed as

$$
h_{i}^{H}(t)=\sum_{\mu=1}^{P} \sigma_{i}^{\mu} m^{\mu}(t)-\alpha \sigma_{i}(t)
$$

Next we consider the the pseudo-inverse rule with synaptic weight matrix expressed in terms of $C^{\mu \nu}$ as

$$
J_{i j}^{P}=\sum_{\mu, \nu=1}^{P} \sigma_{i}^{\mu}\left(C^{-1}\right)^{\mu \nu} \sigma_{j}^{\nu}, i \neq j
$$

It is useful to decompose the state of the network $\sigma_{i}(t)$ in two parts as follows

$$
\sigma_{i}(t)=\sum_{\mu=1}^{P} a^{\mu}(t) \sigma_{i}^{\mu}+\delta \sigma_{i}(t)
$$

where $\delta \sigma_{i}(t)$ is orthogonal to all of the patterns $\left(\sum_{i} \sigma_{i}^{\mu} \delta \sigma_{i}(t)=0\right)$ and $a^{\mu}$ is related to the order parameter $m^{\mu}(t)$ via

$$
a^{\mu}(t)=\sum_{\nu=1}^{P}\left(C^{-1}\right)^{\mu \nu} m^{\nu}(t)
$$

The local field for the pseudo-inverse rule can then be expressed as

$$
\begin{aligned}
h_{i}^{P}(t) & =(1-\alpha) \sum_{\mu=1}^{P} a^{\mu}(t) \sigma_{i}^{\mu}-\alpha \delta \sigma_{i}(t) \\
& =(1-\alpha) \sum_{\mu, \nu=1}^{P} \sigma_{i}^{\mu}\left(C^{-1}\right)^{\mu \nu} m^{\nu}(t)-\alpha \delta \sigma_{i}(t)
\end{aligned}
$$

We can see how Eqn. C14 suppresses the effects of overlaps by considering the state $\sigma_{i}=\sigma_{i}^{1}$. Then $a^{\mu}=\delta_{\mu 1}$ regardless of any correlations of the memories and $h_{i}^{P}=(1-\alpha) \sigma_{i}^{\mu}$. This implies that each memory is an eigenvector of $J_{i j}^{P}$ with eigenvalue $(1-\alpha)$ so that all memories are perfect fixed points for $\alpha \geq 1$. By contrast, we see from Eqn. C10 that 
$h_{i}^{H}$ becomes

$$
h_{i}^{H}=(1-\alpha) \sigma^{1}+\sum_{\mu=2}^{P} \sigma_{i}^{1} m^{\mu}
$$

contains additional noise terms of $O\left(\frac{1}{\sqrt{N}}\right)$ because of overlaps with the other patterns. This noise reduces the capacity of the network as well as the size of the basins of attraction for each memory which are both further reduced if the patterns are not random and contain correlations.

A simplified form of the Storkey rule, discussed in [37, 38, 55], is given by

$$
\begin{aligned}
J_{i j}^{S, 0} & =0 \\
J_{i j}^{S, \mu} & =J_{i j}^{\mu-1}+\frac{1}{N} \sigma_{i}^{\mu} \sigma_{j}^{\mu}-\frac{1}{N} \sigma_{i}^{\mu} h_{j}^{\mu}-\frac{1}{N} h_{i}^{\mu} \sigma_{j}^{\mu}
\end{aligned}
$$

where

$$
h_{i}^{\mu}=\sum_{j=1}^{N} J_{i j}^{\mu-1} \sigma_{j}^{\mu}
$$

Following the analysis in [55] We can find a more compact approximation for $J_{i j}^{S, P}$. We start with $P=1$. Then $J_{i j}^{S, 1}$ is given by

$$
J_{i j}^{S, 1}=\frac{1}{N} \sigma_{i}^{\mu} \sigma_{j}^{\mu}
$$

For $P=2$ we have

$$
J_{i j}^{S, 2}=\sum_{\mu=1}^{2} \frac{1}{N} \sigma_{i}^{\mu} \sigma_{j}^{\mu}-\frac{1}{N^{2}} \sigma_{i}^{2} \sum_{k=1}^{N} \sigma_{j}^{1} \sigma_{k}^{1} \sigma_{k}^{2}-\frac{1}{N^{2}} \sum_{k=1}^{N} \sigma_{i}^{1} \sigma_{k}^{1} \sigma_{k}^{2} \sigma_{j}^{2}
$$

For $P$ memories, keeping terms up to $O\left(\frac{1}{N^{2}}\right), J_{i j}^{S}$ can be expressed as

$$
\begin{aligned}
J_{i j}^{S} & \approx \frac{1}{N} \sum_{\mu=1}^{P} \sigma_{i}^{\mu} \sigma_{j}^{\mu}-\frac{1}{N^{2}} \sum_{\mu, \nu=1}^{P} \sum_{k=1}^{N}\left(1-\delta_{\mu \nu}\right) \sigma_{i}^{\mu} \sigma_{k}^{\mu} \sigma_{k}^{\nu} \sigma_{j}^{\nu} \\
& \approx \frac{1}{N} \sum_{\mu=1}^{P}\left(1+\sum_{k=1}^{N}\left(\sigma_{k}^{\mu}\right)^{2}\right) \sigma_{i}^{\mu} \sigma_{j}^{\mu}-\frac{1}{N} \sum_{\mu, \nu=1}^{P} \sigma_{i}^{\mu}\left(\frac{1}{N} \sum_{k=1}^{N} \sigma_{k}^{\mu} \sigma_{k}^{\nu}\right) \sigma_{j}^{\nu} \\
& \approx \frac{2}{N} \sum_{\mu=1}^{P} \sigma_{i}^{\mu} \sigma_{j}^{\mu}-\frac{1}{N} \sum_{\mu, \nu=1}^{P} \sigma_{i}^{\mu} C^{\mu \nu} \sigma_{j}^{\nu}
\end{aligned}
$$

We can relate $J_{i j}^{S}$ to the Pseudo-inverse rule given in Eqn. C11 by rewriting $J_{i j}^{P}$ using the following series expansion of $C^{-1}$

$$
\begin{aligned}
C^{-1} & =(\mathbb{1}-(\mathbb{1}-C))^{-1} \\
& \approx 2 \mathbb{1}-C+O\left(C^{2}\right)
\end{aligned}
$$

Plugging this expression back into Eqn. C11 gives us

$$
J_{i j}^{P} \approx \frac{1}{N} \sum_{\mu=1}^{P} \sigma_{i}^{\mu}\left(2 \delta^{\mu \nu}-C^{\mu \nu}\right) \sigma_{j}^{\nu}
$$

which is identical to the expression for $J_{i j}^{S}$ given in the last line of Eqn. C20 up to terms of $O\left(\frac{1}{N^{2}}\right)$.

The expansion of $C$ in Eqn. C21 converges if the eigenvalues of $C$ are all contained in the interval [0,2]. For random 

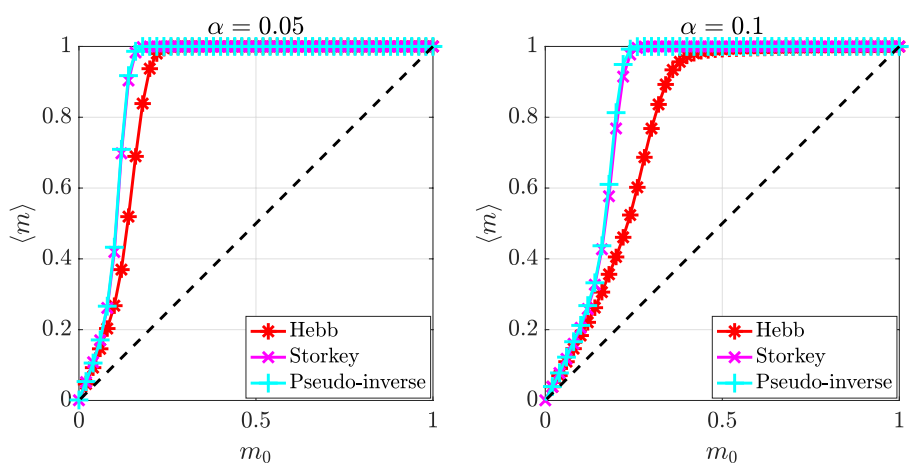

FIG. 12. Comparison of $\langle m\rangle$ as function of retrieval cue overlap $m_{0}$ for the Hebb, Storkey, and Pseudo-inverse learning rules for two values of $\alpha . T=20$ parallel updates, $N=1000$ and 50 trials are used for both figures.

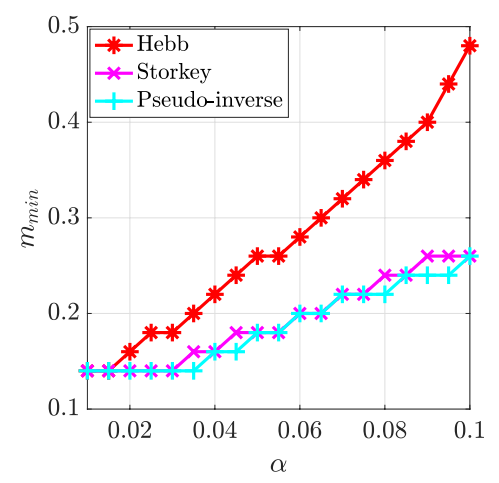

FIG. 13. The average size of the basins of attraction (as defined in the main text) for the Hebb Storkey and Pseudo-inverse learning rules are shown together for small values of $\alpha$.

memories with components $\sigma_{i}^{\mu}$ drawn iid from $\mathcal{N}(0,1)$, the distribution of the eigenvalues of $C^{\mu \nu}$ is given by the Marchenko-Pastur distribution given by, which talks the following form for $P, N \rightarrow \infty$

$$
\rho(\lambda)=\frac{2}{\pi} \frac{\sqrt{\left(\lambda_{+}-\lambda\right)\left(\lambda-\lambda_{-}\right)}}{\lambda}+\mathbb{1}\{\alpha<1\}(1-\alpha) \delta(\lambda), \quad \lambda_{ \pm}=(\sqrt{\alpha} \pm 1)^{2}
$$

Since $\lambda \in\left[\lambda_{-}, \lambda_{+}\right]$, the expansion of $C$ is valid for $\alpha<0.17$ implying that $J_{i j}^{S} \approx J_{i j}^{P}$ for sufficiently small $\alpha$ and large $N$.

In Fig. 12 we compare $\langle m\rangle$ as a function of $m_{0}$ for two values of $\alpha$ for the three learning rules and in Fig. 13, we show $m_{\min }$ (as defined in the main text) as a function of $\alpha$.

\section{Connection to Storage of Knowledge Structures}

While the various learning rules and dynamics have small quantitatively effects on the values on the various network order parameters, their qualitative behavior as a function of the retrieval cue overlap $m_{0}$ remains roughly the same for small values of $\alpha$ below capacity. In all cases, for large $N$ the SNR behaves as

$$
\begin{aligned}
\mathrm{SNR} & \sim \frac{2 c}{\pi} \frac{\langle m\rangle^{2}}{L} \\
& \sim \frac{2 c}{\pi} \frac{f(\alpha)^{2} N m_{0}^{2}}{L} \\
& \sim \frac{8 c}{\pi^{3}} \frac{f(\alpha)^{2} N L_{0}}{L^{2}}
\end{aligned}
$$



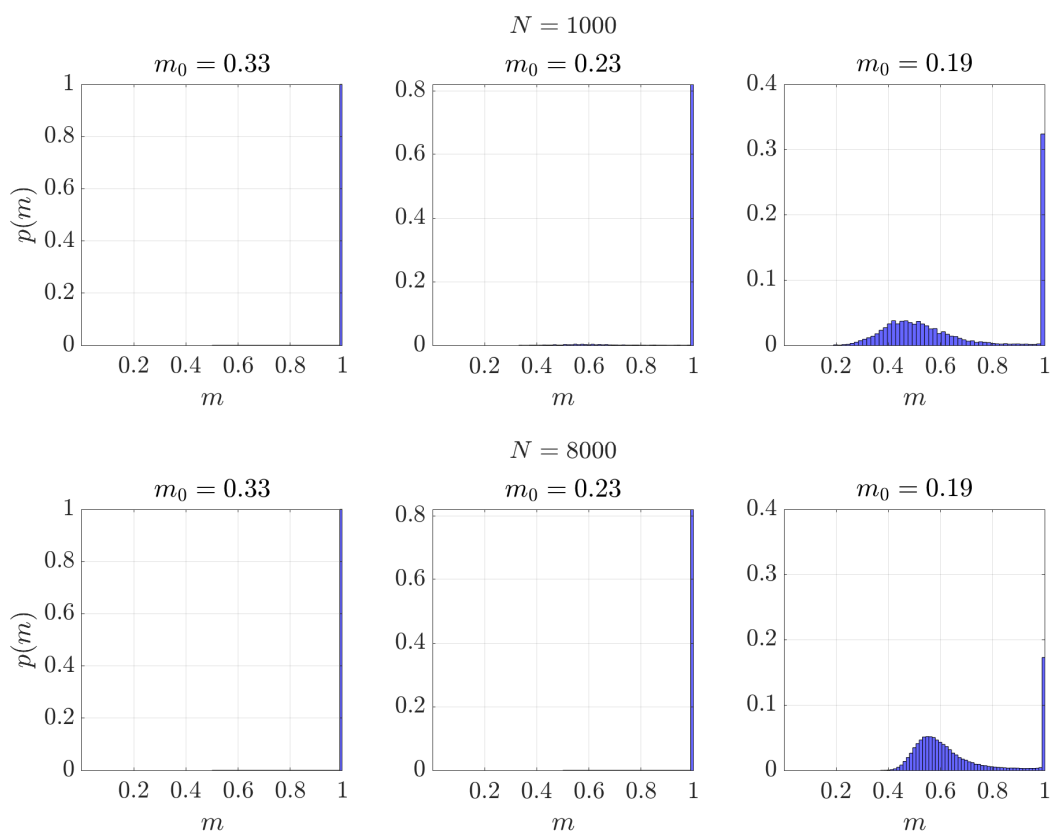

FIG. 14. Histograms for values of $m_{0}$ obtained from Eqn. A40 corresponding to $L_{0}=2$ and $L=8,16,24$ (left to right).

As a result, while the choice of learning rule and retrieval dynamics modify the value of $f(\alpha)$ defined in Eqn. C5 in the main text, they do not change the scaling behavior in Eqn. D1.

\section{Appendix D: Temporal Sequences as Sequences of Attractors}

Previously in $[32,56]$, it was shown that a temporal sequence of memories could be stored in a Hopfield network by adding a second asymmetric synaptic interaction of the form

$$
J_{i j}^{(2)}=\frac{\lambda}{N} \sum_{\ell=1}^{L} \sigma_{i}^{\ell+1} \sigma_{j}^{\ell}, i \neq j
$$

to the synaptic weight matrix, where $L<P$ and $\lambda>1$.

We can store $Q$ sequences in the network by summing together a contribution $J_{i j}^{(2, q)}$ for each sequence, i.e.,

$$
J_{i j}^{(2)}=\sum_{q=1}^{Q} J_{i j}^{(2, q)}
$$

as long as each memory is only contained in one sequence.

To retrieve a sequence, one starts with an initial state $\sigma_{0}=\sigma(0)$ within the basin of attraction of the first memory in the sequence. The evolution of the network state is then given by

$$
\sigma_{i}(t)=\operatorname{sgn}\left(\sum_{j} J_{i j}^{(1)} \sigma_{j}(t-1)+\sum_{j} J_{i j}^{(2)} \bar{\sigma}_{j}(t-1)\right)
$$

where $\bar{\sigma}_{i}(t)$ is defined as

$$
\bar{\sigma}_{i}(t)=\int_{-\infty}^{t} d t^{\prime} w\left(t-t^{\prime}\right) \sigma_{i}(t)
$$

where the function $w(t)$ represents a dynamic memory characterized by time constant $\tau$. A simple choice for $w(t)$ is 
the heaviside function, i.e. $w(t)=\Theta(t)$, which we use for the simulation results shown in Fig. 9D of the main text. $J_{i j}^{(1)}$ is given by a learning rule for the conventional Hopfield network such as the Hebb, Storkey, or Pseudo-inverse rules.

For long sequences, transitions between attractors will be equally spaced with period $t_{0}$ after initial transients and the system will be in the pattern $\mu$ in the time interval $\left((\mu-1) t_{0}, \mu t_{0}\right)$. For a heaviside function $t_{0}$ is given by

$$
t_{0}=\frac{\tau}{2}\left(1+\lambda^{-1}\right)
$$

We can access the performance of this sequence memory by looking at $m^{\mu}(t)$ given in Eqn. C1 for each pattern in a sequence. Good performance requires $m^{\mu}(t) \approx 1$ during the interval $t \in\left((\mu-1) t_{0}, \mu t_{0}\right)$ and $m^{\mu}(t) \ll 1$ for all other $t$.

Note that if a we store two sequences sharing items in the same memory network, transitions between attractors in the dynamics given in Eqn. D3 are no longer unique. 